\title{
Disk Galaxies in the Outer Local Supercluster: Optical CCD Surface Photometry and Distribution of Galaxy Disk Parameters
}

\author{
Nanyao Y. Lu \\ Infrared Processing and Analysis Center \\ MS 100-22, Caltech \\ Pasadena, CA 91125 \\ Email: lu@ipac.caltech.edu
}

\begin{abstract}
We report new B-band CCD surface photometry on a sample of 76 disk galaxies brighter than $B_{T}=14.5 \mathrm{mag}$ in the Uppsala General Catalogue of Galaxies, which are confined within a volume located in the outer part of the Local Supercluster. With our earlier published I-band CCD and high S/N-ratio 21cm HI data (Lu et al. 1993), this paper completes our optical surface photometry campaign on this galaxy sample. As an application of this data set, the B-band photometry is used here to illustrate two selection effects which have been somewhat overlooked in the literature, but which may be important in deriving the distribution function of disk central surface brightness (CSB) of disk galaxies from a diameter or/and flux limited sample: a Malmquist-type bias against disk galaxies with small disk scale lengths (DSL) at a given CSB; and a disk inclination dependent selection effect that may, for example, bias toward inclined disks near the threshold of a diameter limited selection if disks are not completely opaque in optical. Taking into consideration these selection effects, we present a method of constructing a volume sampling function and a way to interpret the derived distribution function of CSB and DSL. Application of this method to our galaxy sample implies that if galaxy disks are optically thin, CSB and DSL may well be correlated in the sense that, up to an inclination-corrected limiting CSB of about $24.5 \mathrm{mag} \operatorname{arcsec}^{-2}$ that is adequately probed by our galaxy sample, the DSL distribution of galaxies with a lower CSB may have a longer tail toward large values unless the distribution of disk galaxies as a function of CSB rises rapidly toward faint values.
\end{abstract}

Subject headings: galaxies: fundamental parameters — galaxies: photometry — galaxies: spiral

\section{Introduction}

Within the Local Supercluster, multi-color CCD surface photometric data are now available on a number of flux-limited samples of disk galaxies in clusters (e.g., Pierce \& Tully 1988; Tully et al. 1996), but the same is not true on field disk galaxies. Lu et al. (1993; hereafter Paper I) selected, in two separate volumes in the Local Supercluster (hereafter LSC), all the disk galaxies brighter than $B_{T} \approx 14.5 \mathrm{mag}$ in the Uppsala General Catalogue of Galaxies (hereafter UGC; Nilson 1973). One of the volumes is toward but beyond our Local Group as viewed from the Virgo Cluster. With a median heliocentric velocity of about $2000 \mathrm{~km} \mathrm{~s}^{-1}$, the UGC sample in this "anti-Virgo Cluster" volume is located in the outer part of the 
Local Supercluster where environmental effects on galaxy disks are probably much less important than in and near the Virgo Cluster, but still close enough to us to have a fairly faint absolute magnitude limit of $M_{B} \sim-17.5$ mag. As a result, this sample is particularly suitable for probing the statistical properties of the disk galaxy population. CCD surface photometry in the I-band and high S/N-ratio $21 \mathrm{~cm}$ HI data on this sample are already published in Paper I. In this paper, we further present new CCD surface photometry in the B-band on this sample.

As an application of this data set, the B-band data are used in the second half of this paper to probe the distribution function of galaxy disk parameters. An exponential disk can be fully described by two fundamental parameters: a central surface brightness (hereafter CSB) and an (exponential) disk scale length (hereafter DSL). How disk galaxies are distributed in terms of these two parameters is important as it may carry information about the physical condition of the universe at the galaxy formation epoch (e.g., Dalcanton et al. 1997a). So far, efforts have been mostly in determining this distribution function partially integrated over DSL, namely, the CSB distribution. Freeman (1970) showed that the CSBs of disks of a sample of local disk galaxies are distributed in a narrow range of $21.6 \pm 0.3 \mathrm{mag} \operatorname{arcsec}^{-2}$. This so-called Freeman law was later interpreted by Disney (1976) and Disney \& Phillipps (1983) to be a selection effect due to the fact that a sample selected on the basis of a limiting diameter at a fixed surface brightness (hereafter SB) may miss giant disk galaxies (with a large DSL) whose CSBs are too faint, as well as compact galaxies (with a bright CSB) whose DSLs are too small. Note that the same selection effect could also occur in a flux limited sample.

The existence of the above selection effect in optical galaxy catalogs such as UGC has been supported by a large number of studies (e.g., Allen \& Shu 1979; Davies 1990; Schombert et al. 1992; McGaugh et al. 1995; McGaugh 1996; de Jong 1996; Sprayberry et al. 1996; Dalcanton et al. 1997b; and the references in Bothun et al. 1997). But there is still considerable controversy about the exact shape of the CSB distribution for relatively bright disk galaxies. For example, using a diameter-limited galaxy sample and a volume sampling function based on both CSB and DSL, van der Kruit (1987) concluded that after excluding dwarf galaxies, there are not many large, low-CSB disk galaxies; on the other hand, using larger galaxy samples and a volume sampling function based on CSB alone, other groups have derived CSB distributions that are nearly flat at values fainter than the canonical Freeman's value of B 21.6 mag arcsec ${ }^{-2}$ (e.g., Davies 1990; McGaugh et al. 1995; McGaugh 1996). For a further discussion on this controversial subject, see Briggs (1997).

There are, however, a number of issues which have not been formally addressed in the past and which may be important to correctly interpreting any CSB distribution function derived from a diameter or flux limited sample. The first one concerns the so-called Malmquist bias (Malmquist 1920). At a given CSB, the DSL distribution is fairly wide (e.g., McGaugh et al. 1995, de Jong 1996). A diameter (or flux) limited selection leads to a Malmquist-type bias in the sense that one tends to select only intrinsically large disks at a given CSB. Near the limiting SB of a sample selection, a slight dimming in CSB has to be compensated by a large increase in DSL in order for a galaxy to be selected. Therefore, a bias could occur between highand low-CSB disk galaxies.

The second issue is how to take into account the effect of disk inclination on the detectability of a disk galaxy. Depending on how transparent a galaxy disk is in optical, this effect could be particularly important to galaxies with disk parameters near the selection threshold. As we show in this paper, depending on whether disks are opaque or transparent, the same galaxy sample could lead to a quite different conclusion on the derived distribution function of galaxy disk parameters. 
The third issue regards whether the variables, CSB and DSL, are separable in the bi-variate galaxy distribution function. In other words, are CSB and DSL statistically independent of each other? A positive answer to this question would allow one to derive a CSB distribution function from a diameter or flux limited sample without requiring complete redshift data. Some authors have argued for and used such a statistical independence between CSB and DSL (e.g., McGaugh 1996), but this has never been rigorously tested. A possible correlation between the two parameters is hinted by theoretical considerations (e.g., Dalcanton 1997a) and by the observational fact that low-CSB spiral galaxies tend to have a large DSL (e.g., Kent 1985; Bothun et al. 1990; Dalcanton et al. 1997b). When examining the apparent DSL distribution of galaxies from a diameter or flux limited sample, one has to be aware of the Malmquist bias: at fixed CSB and distance, the bias prevents one from sampling galaxies with a DSL shorter than some threshold. Without a prior knowledge of the intrinsic DSL distribution function, the only secure measurement one can make is on the part of the DSL distribution above this threshold. Therefore, for a set of well defined selection criteria, and as we show in this paper, one can still answer the question as to whether the DSL distribution at large values depends on CSB.

The remainder of this paper is organized as follows: In Sect. 2, we describe the galaxy sample, our B-band CCD surface photometry and present the photometric results. In Sect. 3, we illustrate the two selection effects introduced above; and by taking into consideration these selection effects, present a method of constructing a volume sampling function and a way to interpret the derived distribution function of disk parameters. The method is then applied to our galaxy sample. In Sect. 4, we discuss some implications from our analyses. We end this paper with a brief summary in Sect. 5. Throughout this paper, we assume a Hubble constant of $75 \mathrm{~km} \mathrm{~s}^{-1} \mathrm{Mpc}^{-1}$, and use the following notations for an exponential disk: $\mu_{0}\left(\mu_{0}^{c}\right)$ for its observed (face-on) CSB in units of mag $\operatorname{arcsec}^{-2}$ and $r_{s}\left(h_{s}\right)$ for its angular (linear) DSL in units of $\operatorname{arcsec}(\mathrm{kpc})$. Thus, an exponential disk can be expressed as

$$
\mu(r)=\mu_{0}+1.086\left(r / r_{s}\right) .
$$

\section{B-Band CCD Surface Photometry}

\subsection{Observations}

Our galaxy sample, originally selected in Paper I and used in Lu et al. (1994) to study the local velocity field, contains all the 76 UGC disk galaxies with $B_{T}<14.5$ mag within a volume bounded by $22^{\mathrm{h}}<\alpha<2^{\mathrm{h}}$, $0^{\circ}<\delta<20^{\circ}$ and a heliocentric velocity of $0<v_{h}<3000 \mathrm{~km} \mathrm{~s}^{-1}$. Our B-band CCD observations were carried out with the Hale 200" telescope equipped with the four shooter (Gunn et al. 1987) at Palomar Observatory from August 25 to 27, 1990 (UT) under a photometric condition. We used the 4-shooter's standard violet filter $(4300 \AA / 700 \AA)$ to mimic Johnson's B system (Johnson \& Morgan 1953). The resulting CCD field is a $4^{\prime} .4$ square with a pixel size of $0 .{ }^{\prime \prime} 336$. In addition to this UGC sample, we also observed a number of optically fainter galaxies as described in Paper I (also see Hoffman et al. 1996). The integration time per galaxy ranges from 4 to 8 minutes. The data reduction procedure is similar to that in Paper I. The final images ready for surface photometric analysis show a quite flat background. For example, the mode and mean of sky pixels usually agree with each other within $0.3 \%$. The instrumental magnitudes were converted into Johnson B system using the observed standard stars taken from Landolt (1983). 


\subsection{Surface Photometry}

Surface photometric analysis was done by fitting elliptical contours to each sky-subtracted galaxy image following the prescription given in Paper I. The fitting was performed on the B-band images, independent of the existing I-band results in Paper I. In most cases, a good fit could be obtained down to a SB of B $26 \mathrm{mag} \mathrm{arcsec}^{-2}$. For each galaxy, the resulting SB profile as a function of the semi-major axis, $r$, was displayed and its outer part between two radii $r_{1}$ and $r_{2}$, dominated by the disk component as judged by eyes, was fit into eq. (1). The mean ellipticity of the disk component, $e$, was evaluated between the radii $r_{1}$ and $r_{2}$. The isophotal magnitude, $B_{26}$, and diameter, $D_{26}$, were measured at B $26 \mathrm{mag} \mathrm{arcsec}^{-2}$ isophote determined by the fitted exponential disk profile. For a number of galaxies whose B 26 mag $\operatorname{arcsec}^{-2}$ isophotes are partially outside the CCD field, the exponential disk fit was used in each case to evaluate the contribution to $B_{26}$ from those isophotes partially outside the CCD field. Finally, the total magnitude, $B_{\text {tot }}$, was evaluated by extrapolating the isophotal magnitude at $r_{2}$ to infinity in radius using the exponential disk fit.

We list in Table 1 all the 76 UGC sample galaxies as well as those optically fainter ones that we observed. Of the 76 UGC sample galaxies, seven do not have photometric data for various reasons as given at the end of Table 1. The table columns are as follows: Col. (1) is the galaxy name as in UGC, but for those fainter galaxies we give their names as in Paper I. Col. (2) gives the NGC or IC number if applicable. The adopted distance in $\mathrm{Mpc}$ is given in col. (3), derived from the velocity of the galaxy with respect to the centroid of the LG [i.e., $v_{h}+300 \sin (l) \cos (b)$ ]. Cols. (4) and (5) are $r_{1}$ and $r_{2}$ in arcsec, respectively; namely, the inner and outer radii for the exponential disk fit. Col. (6) is the mean disk ellipticity, $e$, which has been used to derive the disk inclination angle in degrees in col. (7) via

$$
\cos ^{2}(i)= \begin{cases}\frac{(1-e)^{2}-0.2^{2}}{1-0.2^{2}}, & \text { if } e \leq 0.8^{\circ} \\ 0, & \text { otherwise. }\end{cases}
$$

Col. (8) is the mean position angle of the disk on the sky ( $\mathrm{N}$ to E) measured between the radii $r_{1}$ and $r_{2}$. Cols. (9) and (10) are respectively $B_{26}$ in mag and $D_{26}$ in arcmin. Col. (11) is the angular DSL in arcsec and col. (12) the linear DSL in kpc. Col. (13) is $\mu_{0}$ in mag $\operatorname{arcsec}^{-2}$ determined from the exponential disk fit. Col. (14) is the total magnitude $B_{\text {tot }}$. Col. (15) is the B-band absolute magnitude derived from $B_{t o t}$ and the distance in col. (3). Finally, Col. (16) gives $(B-I)$ color derived from $B_{\text {tot }}$ in this paper and $I_{\text {tot }}$ in Paper I. No correction for Galactic or internal reddening has been applied to the parameters in Table 1.

Fig. 1 displays the observed B-band surface brightness as a function of the semi-major axis for each of the galaxies with photometric parameters in Table 1. The open squares represent the measured isophotes, while the filled square represents the fitted isophote at B 26 mag arcsec ${ }^{-2}$.

\subsection{Uncertainties and Systematics}

A number of galaxies were observed multiple times over the entire observing run. The multiple images of the same galaxy were reduced independently from each other and the results are used as a way to measure the statistical uncertainties in the derived photometric parameters. Such estimated r.m.s. uncertainties are on the order of $0.01 \mathrm{mag}$ for $B_{26}, 0.05 \mathrm{mag}$ for $B_{\text {tot }}, 3^{\circ}$ for the disk inclination angle, $0.3 \mathrm{mag} \operatorname{arcsec}^{-2}$ for

$\mu_{0}$, and $7 \%$ for $r_{s}$. Another way to illustrate our photometric accuracy is to compare the B-band result here with the I-band result in Paper I on the same galaxy. As an example, plotted in Fig. 2 as functions of the B-band disk ellipticity are the differences in the measured disk position angle (P.A.) and ellipticity between 
the two bandpasses. As expected, the more inclined a galaxy disk is, the better agreement between the two bandpasses is in Fig. 2. For galaxies inclined more than $45^{\circ}(e \sim 0.3)$, the typical agreement between the two bandpasses is within $\sim 5^{\circ}$ in P.A. and $\sim 10 \%$ in disk ellipticity (or $\sim 2.5^{\circ}$ in terms of disk inclination angle).

Our $B_{\text {tot }}$ magnitudes are however fainter by about $0.06 \mathrm{mag}$ on average than the $B_{T}$ magnitude scale of the Third Reference Catalogue of Galaxies (RC3; de Vaucouleurs et al. 1991). This is illustrated in Fig. 3. A Gaussian curve, with a center at $\left(B_{\text {tot }}-B_{T}\right)=0.06$ mag and a FWHM of 0.4 mag, is shown in the figure for comparison. No obvious correlation could be identified between $\left(B_{\text {tot }}-B_{T}\right)$ and the night on which $B_{\text {tot }}$ was obtained, galaxy morphology, optical color or $B_{T}$.

It is also interesting to see how the fraction of light outside the B $26 \mathrm{mag} \operatorname{arcsec}^{-2}$ isophote vary with the disk central surface brightness. Fig. 4 shows that $\left(B_{26}-B_{\text {tot }}\right)$ increases as $\mu_{0}$ increases. Note that, for a low-SB galaxy of $\mu_{0} \gtrsim 24 \mathrm{mag} \operatorname{arcsec}^{-2}$, more than half of its luminosity lies outside the B $26 \mathrm{mag} \mathrm{arcsec}^{-2}$ isophote.

Because we did not do a full bulge/disk decomposition, the CSB of a galaxy with a prominent bulge could be overestimated (Kormendy 1977). We found however that the eraly-type disk galaxies do not show on average a brighter CSB than those late-type galaxies, suggesting that our disk fitting procedure is probably insignificantly affected by the size of a galactic bulge. On the other hand, there are a number of sample galaxies with prominent spiral arms forming a ring-like pattern. These include UGC 12343, UGC 12447, UGC 12777, and UGC 00858. For each of these galaxies, the SB profile outside the spiral arms, where we have fit its exponential disk, radially falls off fairly quickly to a faint level. The resulting CSBs of these galaxies are among the brightest in the sample. Should we have fit an exponential disk to the entire galaxy surface, we would have obtained a fainter CSB in each galaxy. It is not clear which way is better. But not all high-SB sample galaxies are of this type. For example, UGC 12074, UGC 12529 and UGC 00167 are also among those of the brightest CSBs in the sample, but none of them show prominent spiral arms in optical. In fact, with $18.3<\mu_{0}<19.5 \mathrm{mag} \mathrm{arcsec}^{-2}$ and a moderate disk inclination, these 3 disk galaxies may represent a class of relatively rare, "super high-SB" disk galaxies. We will study these three galaxies in more details in a future paper.

\section{On the Disk Parameter Distribution Function}

\subsection{Formulation of the Sample Selection}

As a conventional simplification, we formulate the UGC sample selection by assuming a negligible effect from the bulge of a galaxy. This is probably a reasonable simplification as most of our sample galaxies are dominated by their disks. We define an intrinsic or face-on CSB as follows:

$$
\mu_{0}^{c}= \begin{cases}\mu_{0}-2.5 K \log (1-e), & \text { if } e \leq 0.8^{\circ} \\ \mu_{0}-2.5 K \log (1-0.8), & \text { othersie. }\end{cases}
$$

The transition at $e=0.8$ in eq. (3) is chosen somewhat arbitrarily. It corresponds to the onset of $i=90^{\circ}$ when the disk inclination angle $i$ is given by eq. (2). The value of $K$ is limited to $0 \leq K \leq 1$, with the lower limit corresponding to a completely opaque disk and the upper limit to a fully transparent disk. In spite of extensive studies on the opacities of galaxy disks, it is still highly controversial as to whether galaxy disks are largely opaque or transparent (e.g., Tully \& Fouqué 1985; Disney, Davies, \& Phillipps 1989; Valentijn 1990, 1994; Burstein, Haynes, \& Faber 1991; Byun 1993; Giovanelli et al. 1994; Xu \& Buat 1995; Tully \& 
Verheijen 1997). In this paper we consider only two cases: (a) fully transparent disks with $K=1.0$ and (b) fairly opaque disks with $K=0.2$.

We plot in Fig. $5 \mu_{0}$ as a function of $r_{s}$ for the 69 UGC sample galaxies with B-band photometry. The filled and open squares represent those with a disk ellipticity below and above 0.5 , respectively. It is clear that, at a given $r_{s}$, disks of larger inclination angles are on average associated with brighter values of $\mu_{0}$ [cf. inequality (6) below]. The solid curve in the figure represents the selection limit associated with the UGC limiting diameter of $1^{\prime}$ at $\mu_{B} \approx 25.3 \mathrm{mag} \operatorname{arcsec}^{-2}$ (Cornell et al. 1987) as follows:

$$
\mu_{0} \leq 25.3-1.086\left(30^{\prime \prime} / r_{s}\right) \text {. }
$$

For an exponential disk, its total magnitude can be written as

$$
B_{T}=\mu_{0}-5 \log r_{s}-2.5 \log (1-e)-1.995 .
$$

Our magnitude selection criterion of $B_{T} \leq 14.5 \mathrm{mag}$ transfers to

$$
\mu_{0} \leq 5 \log \left(r_{s}\right)+2.5 \log (1-e)+16.49
$$

Note that as in eq. (3), we simply set $e=0.8$ in both criteria (5) and (6) for cases of $e>0.8$. It is clear that only for a fully transparent disk, is criterion (5) independent of $e$. We plot criterion (6) in Fig. 5 for the cases of $e=0$ and $e=0.8$ by the dotted and dashed curves, respectively.

To have a rough, but quantitative picture of the overall sample selection, we give in Table 2 a few numerical indicators on how our sample selection acts on face-on disks of a given CSB: Column (2) is the minimum angular DSL for a galaxy of a given CSB to be selected. This is given by combining criteria (4) and (6). Column (3) gives $\Gamma(-17.5) / \Gamma_{\max }^{s}$, where $\Gamma_{\max }^{s}$ is the sample distance cutoff and is taken to be 42.5 Mpc in this paper, and $\Gamma(-17.5)$ is the maximum distance at which a galaxy of $M_{B}=-17.5 \mathrm{mag}$ can still be selected. This quantity is given by relating the minimum $r_{s}$ in column (2) to the following relation on the absolute magnitude in the case of $M_{B}=-17.5 \mathrm{mag}$ and $e=0$,

$$
M_{B}=\mu_{0}-5 \log \left(h_{s}\right)-2.5 \log (1-e)-38.57 .
$$

Column (3) shows that a disk galaxy of $M_{B}=-17.5$ mag can be seen up to about half of $\Gamma_{\max }^{s}$ in distance for the most part of the CSB range explored here. So roughly speaking, the part of the galaxy population with $M_{B}<-17.5 \mathrm{mag}$ is adequately sampled here. Finally, columns (4) and (5) are $h_{s}(-17.5)$ and $h_{s}\left(\Gamma_{\max }^{s}\right)$, respectively, where $h_{s}(-17.5)$ is the linear DSL of a galaxy of $M_{B}=-17.5$ mag via eq. (7) and $h_{s}\left(\Gamma_{\max }^{s}\right)$ is the minimum $h_{s}$ that a disk galaxy has to have in order to be selected out to the sample cutoff distance $\Gamma_{\max }^{s}$.

\subsection{Illustration of a Malmquist Bias}

The presence of a Malmquist bias in our sample is illustrated in Fig. 6, where we plot $\mu_{0}^{c}$ as function of $h_{s}$ for the sample galaxies in two cases: (a) fully transparent disks with $K=1.0$, and (b) fairly opaque disks with $K=0.2$. The dotted and solid curves in the figure are generated by using columns (4) and (5) of Table 2, respectively. Clearly, the distribution pattern of the data points in each plot suggests that at a given CSB, only galaxies with large enough $h_{s}$ have been selected. This bias becomes progressively severe when the CSB under consideration approaches the limiting SB of the sample selection. Without taking into consideration this bias, a distribution of CSB or DSL derived from our UGC sample would also be biased. 
Unfortunately, one usually does not have a prior knowledge about the intrinsic shape of the $h_{s}$ distribution, especially at a faint CSB level, a correction for this Malmquist bias remains model dependent at best.

Although this Malmquist bias makes it impossible to use our sample to conduct a full bi-variate analysis of the galaxy distribution, we can still determine, at a given CSB, the part of the $h_{s}$ distribution that is adequately sampled by our sample. Roughly speaking, this is the region to the right of each dotted curve in Fig. 7.

\subsection{Derivation of a Volume Sampling Function}

Let us consider a galaxy of an exponential disk with fixed $\mu_{0}^{c}$ and $h_{s}$. The corresponding observables are $\mu_{0}$ and $r_{s}$, respectively. Such a galaxy would be selected if its distance $\Gamma$, which relates $h_{s}$ to $r_{s}$, and inclination angle $i$, which relates $\mu_{0}^{c}$ to $\mu_{0}$ via eq. (3), are such that both criteria (4) and (6) are satisfied. We sketch this in Fig. 7 for both the cases of $K=1.0$ and $K=0.2$, with the help from the same curves as shown in Fig. 5. For a fully transparent disk with $K=1.0$, at a given $r_{s}$, an increasing disk inclination would move the galaxy vertically upward, as illustrated by the thick arrow, from the horizontal line marked as " $i=0^{\circ}$ " to the one marked as " $i \geq 80^{\circ}(\mathrm{K}=1.0)$." This remains true as long as $r_{s}$ is greater than that of the vertical line " $\mathrm{a}-\mathrm{b}_{1}$ " in the figure, which marks the farthest point in distance at which this galaxy can still be selected in our UGC sample. For a fairly opaque disk with $K=0.2$, at a large value of $r_{s}$, an increasing disk inclination still moves the galaxy vertically upward, but only to the horizontal line marked as " $i \geq 80^{\circ}(\mathrm{K}=0.2)$." The situation changes when the $r_{s}$ value of the galaxy becomes smaller than that of point "b2" in Fig. 7. When this happens, at each $r_{s}$, the galaxy can move vertically up to the line "a-b $b_{2}$ " as illustrated by the thin arrow in Fig. 7. In other words, the galaxy would be selected only if its disk inclination is small enough, and at point "a" the galaxy would be included in our UGC sample only if its disk is viewed face-on. To summarize, the detectability of an optically thin disk is much independent of its inclination, while a fairly opaque disk could be selected farther in distance at smaller disk inclination angle. This statement needs a slight modification when the solid curve surpasses both the dashed and dotted curves in Fig. 7 and becomes the most stringent selection criterion, i.e., at $\mu_{0}<18 \mathrm{mag} \mathrm{arcsec}^{-2}$ or $\mu_{0} \gtrsim 25 \mathrm{mag}_{\operatorname{arcsec}}^{-2}$. But as evident in Fig. 5, few sample galaxies are in these regimes.

We now incorporate this inclination dependent detectability into a volume sampling function. For a given galaxy at a given distance $\Gamma$ (or $r_{s}$ ), one can define $V_{\text {max }}^{i}$, the maximum "volume" in the phase space of the disk inclination angle, to be

$$
V_{\max }^{i}=\left(i_{\max }-i_{\min }\right) / 90^{\circ},
$$

where $i_{\min }$ and $i_{\max }$, both in degrees, are the minimum and maximum possible values for the inclination angle of this galaxy as described above. $i_{\min }$ can be either zero or greater. For $K=1.0, i_{\max }$ always equals $90^{\circ}$. For $K<1, i_{\max }$ could be either $90^{\circ}$ or smaller.

Next we let the distance of the galaxy vary (so does $r_{s}$ ), both $i_{\min }$ and $i_{\max }$ are now functions of the distance of the galaxy. Denote $\Gamma_{\text {max }}^{i}$ as the maximum distance a galaxy can still be detected when it is at the most favorable disk inclination (e.g., point "a" in Fig. 7), we can define a composite maximum (space + inclination) phase volume as follows

$$
V_{\max }=\int_{0}^{\min \left(\Gamma_{\max }^{i}, \Gamma_{\max }^{s}\right)} V_{\max }^{i} \Omega \Gamma^{2} d \Gamma
$$

where $\Omega$ is the constant solid angle $(\approx 0.13 \mathrm{sr})$ of our sample on the sky, and $\Gamma_{\max }^{s}(=42.5 \mathrm{Mpc})$ is our 
sample distance cutoff.

Let $n\left(\mu_{0}^{c}, h_{s}\right)$ be the space density distribution function of disk galaxies in terms of CSB and DSL, in the absence of the Malmquist bias, one would have

$$
n\left(\mu_{0}^{c}, h_{s}\right) \Delta \mu_{0}^{c} \Delta h_{s}=\Sigma\left(1 / V_{\max }\right),
$$

where the sum is over all the sample galaxies with $\mu_{0}^{c}$ and $h_{s}$ within the intervals $\Delta \mu_{0}^{c}$ and $\Delta h_{s}$, respectively.

\subsection{Results from our UGC Galaxy Sample}

We have calculated $V_{\max }$ for each of the 69 UGC sample galaxies with the B photometry in Table 1 . Although we left out the other 7 sample galaxies because of their unavailable B photometry, this should not have a significant effect on the shape of our derived distribution function. The Malmquist bias and our moderate sample size prevent us from using eq. (10) directly. Instead, we divide the UGC sample into 4 consecutive bins in the inclination corrected CSB and derive a DSL distribution within each of these bins. The results are shown in Fig. 8 for the case of $K=1.0$ and in Fig. 9 for the case of $K=0.2$. One galaxy (UGC 01466) with $h_{s} \approx 12 \mathrm{kpc}$ and a relative density of $\sim 0.04$ for the bin of $21.5<\mu_{0}^{c}<23.0 \mathrm{mag} \mathrm{arcsec}^{-2}$ is off the figure. As noted in Table 1, the disk fit of this galaxy was performed over a range of radii dominated by the prominent spiral arms of the galaxy. It is likely that its DSL has been overestimated. A total of 10 sample galaxies with $M_{B}>-17.5 \mathrm{mag}$ have also been excluded from these figures. Should they be included here, most of them would occupy the region in each CSB bin to the left of the arrow which roughly indicates the threshold in $h_{s}$ below which the Malmquist bias makes the distribution incomplete (see Fig. 6 or Table 2). The part of the distribution to the right of the arrow is considered here to represent the true DSL distribution subject to the statistical error. It is this part of the distribution we use to draw the following results.

Let us concentrate on the two faintest, equally wide CSB bins in these figures, namely, (i) $21.5<\mu_{0}^{c}<23.0 \mathrm{mag} \operatorname{arcsec}^{-2}$ and (ii) $23.0<\mu_{0}^{c}<24.5 \mathrm{mag} \mathrm{arcsec}^{-2}$. These bins cover the flat part of the CSB distribution shown, for example, in McGaugh (1996). In the case of transparent disks with $K=1.0$ (see Fig. 8), the distribution in the fainter CSB bin (ii) has a longer tail toward large $h_{s}$ values. For example, the integrated density over $h_{s}>5 \mathrm{kpc}$ is about $0.41( \pm 0.17)$ in bin (ii). Note that all the galaxies with $h_{s}>5 \mathrm{kpc}$ in bin (i) would be detectable up to the maximum sample distance (cf. Table 2). Should the CSB distribution be nearly flat with CSB and DSL being statistically independent of each other (e.g., McGaugh 1996), one would expect to see about $11( \pm 4)\left[\approx 0.41 \times(1 / 3) \times\left(\Gamma_{\max }^{s} / 10 \mathrm{Mpc}\right)^{3}\right]$ galaxies with $h_{s}>5 \mathrm{kpc}$ in bin (i). But we actually observed only one galaxy. Thus, at a significance level of $2.5 \sigma$, our analysis suggests either (a) that CSB and DSL are correlated in the sense that the DSL distribution at a fainter CSB level has a longer tail toward large values; or (b) that CSB and DSL are still independent of each other, but with a CSB distribution function that increases rapidly toward faint CSB values (i.e., at a rate 10 times faster than a flat CSB distribution).

In the case of fairly opaque disks with $K=0.2$ as shown in Fig. 9, we have only marginally useful data in the faintest CSB bin (ii). Under the assumptions of a nearly flat CSB distribution and a statistical independence between CSB and DSL, the same analysis as above implies that the expected number of

galaxies with $h_{s}>5 \mathrm{kpc}$ in the CSB bin (i) is $5( \pm 3)$. We actually observed 5 galaxies in that bin. However, this comparison is only significant at $1.5 \sigma$. 


\section{Discussion}

The two selection effects that we discussed and formulated in the previous section, namely, a Malmquist bias and a disk inclination dependent detectability, should be present in any diameter or/and flux limited sample. As we have shown here, both of these could have a significant effect on how to interpret a distribution function derived from a diameter/magnitude limited sample. We note that neither the visibility theory of Disney and Phillipps (1983) nor the volume sampling function of McGaugh (1996) has fully addressed these selection effects.

For a diameter limited sample, the volume sampling function is proportional to $h_{s}^{3}\left(\mu_{\text {limit }}-\mu_{0}\right)^{3}$ (McGaugh 1996), where $\mu_{\text {limit }}$ is the limiting SB in the sample selection. This sensitive dependence on $h_{s}$ makes the Malmquist bias particularly severe near the limiting SB of a sample selection. For example, our Fig. 6 shows that $h_{s}^{\text {min }}$, the threshold in $h_{s}$ below which our UGC sample is highly incomplete, increases from about $1.5 \mathrm{kpc}$ for the range of $21.5<\mu_{0}^{c}<23 \mathrm{mag} \mathrm{arcsec}^{-2}$ to $3.5 \mathrm{kpc}$ for $23<\mu_{0}^{c}<24.5 \mathrm{mag} \mathrm{arcsec}^{-2}$. This Malmquist bias, if not corrected for, has the following implications: If the full volume sampling function as defined above is used (see an example in van der Kruit 1987), one will always overestimate the mean volume sampling function for the population of the low-CSB galaxies relative to that of the high-CSB galaxies, leading to a relative underestimate of the galaxy number density at low CSB values. On the other hand, if a volume sampling function that scales only with $\left(\mu_{\text {limit }}-\mu_{0}\right)^{3}$ is used (see an example in McGaugh 1996), one will relatively underestimate the volume sampling functions for the low-CSB galaxies in the sample. Although this underestimate works in favor of compensating the Malmquist bias, it is no guarantee that the compensation would work out perfectly.

To better understand how disk parameters are distributed at the low SB end and to effectively constrain disk formation models such as that proposed by Dalcanton et al. (1997a), we need galaxy samples selected with a small limiting diameter at a faint SB. Unfortunately, this implies that the resulting catalog might be too large for achieving a completeness in redshift. One alternative way is to use some cluster sample as illustrated in Tully \& Verheijen (1997), for which complete redshift data are not needed (except for weeding out field galaxy contaminations). Note that the selection effects discussed in this paper still apply to a cluster sample. A good example of this is shown in Fig. 4 of Dalcanton et al. (1997a) on a complete sample of Virgo Cluster galaxies. A systematic HI survey (e.g., Szomoru et al. 1994) with follow-up optical CCD photometry on the detected galaxies is another alternative approach that could offer an unbiased picture on how galaxy disk parameters distribute above a certain threshold in HI mass.

Although the shape of the CSB distribution function is still quite uncertain at faint SB levels, it is rather clear from this study and those cited in Sect. 1 that this distribution function is fairly wide over CSB even for bright disk galaxies, extending to much fainter values than the narrow range initially proposed by Freeman. However, our data (see Figs. 5 and 6 ) do not show a clear support for a bimodal CSB distribution. This is further confirmed by our I-band data from Paper I in which disk internal reddening is only about $40 \%$ of that in the B-band. A bimodal CSB distribution is observed on disk galaxies in the Ursa Major cluster in both I and K (Tully \& Verheijen 1997). It is not clear at this point if this difference implies that the bimodal CSB distribution is a phenomenon specific to certain clusters.

\section{Summary}

In this paper, we present new B-band CCD surface photometry on (1) 69 galaxies in a complete sample of 76 disk galaxies brighter than $B_{T}=14.5 \mathrm{mag}$ in the Uppsala General Catalogue of Galaxies and (2) 11 
additional galaxies that are optically fainter than 14.5 mag. Surface brightness and color radial profiles are shown and various photometric parameters are tabulated on each of these galaxies. This data set complements our earlier published I-band CCD and high S/N-ratio $21 \mathrm{~cm}$ HI data on the same galaxies (Lu et al. 1993).

The B-band data are then used to study the distribution of the fundamental galaxy disk parameters: the central surface brightness (CSB) and (exponential) disk scale lengths (DSL). We illustrate two selection effects that occur in any diameter or/and flux selected sample of disk galaxies: (1) there is always a Malmquist-type selection effect that biases against disk galaxies with small disk scale lengths at a given CSB, and (2) there could be a dependence of the detectability of a galaxy on it disk inclination angle as long as disks are not completely opaque. Without a prior knowledge on the full distribution function of the disk parameters, it is difficult to fully correct for the Malmquist bias. On the other hand, we derive a volume sampling function that takes into account the inclination effect.

Using this volume sampling function, we derive a relative density distribution of DSL for a given range of CSB values from the UGC sample for each of the following two cases: (a) fully transparent disks and (b) fairly opaque disks. Replying on only the part of the resulting distribution function that is least affected by the Malmquist bias, we show that in the case of (a), CSB and DSL could be correlated in the sense that, up to an inclination-corrected limiting CSB of about $24.5 \mathrm{mag} \operatorname{arcsec}^{-2}$ adequately sampled by our galaxy sample, the DSL distribution of galaxies with a lower CSB may have a longer tail toward large values unless the distribution of disk galaxies as a function of CSB rises very rapidly toward faint values. In the case of (b), the face-on limiting CSB of our galaxy sample is too faint to set a useful constraint on the faint part of the CSB distribution function.

The author is grateful to E. E. Salpeter and G. L. Hoffman for their comments on this paper and contribution to the early part of our CCD surface photometry campaign; to the editor, G. Bothun, and the referee, S. McGaugh, for a number of comments that helped improving this paper; to W. Freudling for providing us with the GALPHOT software package which is part of the data reduction tools used in this paper; to M. Schmitz for going over the tables; and to the staff members of Palomar Observatory for their assistance during the observation. The observations presented in this paper were made at the Palomar Observatory as part of a continuing collaborative agreement between the California Institute of Technology and Cornell University. This work was supported in part by Jet Propulsion Laboratory, California Institute of Technology, under a contract with the National Aeronautics and Space Administration.

\section{REFERENCES}

Allen, R. J., \& Shu, F. H. 1979, ApJ, 227, 67

Bothun, G. D., Schombert, J. M., Impey, C. D., \& Schneider, S. T. 1990, ApJ, 360, 427

Bothun, G., Impey, C., and McGaugh, S. 1997, PASP, 109, 745

Briggs, F. H. 1997, ApJ, 484, L29

Burstein, D., Haynes, M. P., \& Faber, S. M. 1991, Nature, 353, 515

Byun, Y.-I. 1993, PASP, 105, 993

Cornell, M. E., Aaronson, M., Bothun, G., \& Mould, J. 1987, ApJS, 64, 507 
Dalcanton, J. J., Spergel, D. N., \& Summers, F. J. 1997a, ApJ, 482, 659

Dalcanton, J. J., Spergel, D. N., Gunn, J. E., Schmidt, M., \& Schneider, D. P. 1997b, AJ, 114, 635

Davies, J. I. 1990 MNRAS, 244, 8

de Jong, R. S. 1996, A\&A, 313, 45

de Vaucouleurs, G., de Vaucouleurs, A., Corwin, H. G., Buta, R. J., Paturel, G., \& Foueué, P. 1991, Third Reference Catalogue of Bright Galaxies (New York: Springer) (RC3)

Disney, M. J. 1976, Nature, 263, 573

Disney, M. J., \& Phillipps, S. 1983, MNRAS, 205, 1253

Disney, M. J., Davies, J., \& Phillipps, S. 1989, MNRAS, 239, 939

Freeman, K. C. 1970, ApJ, 160, 811

Giovanelli, R., Haynes, M. P., Salzer, J. J., Wegner, G., Costa, L. N., Freudling, W. 1994, AJ, 107, 2036

Gunn, J. E., et al. 1987, Opt. Engineering, 26, 779

Hoffman, G. L., Dickey, J., Lu, N. Y., \& Fromhold-Treu, R. 1996, ApJ, 473, 822

Johnson, H. L., \& Morgan, W. W. 1953, ApJ, 117, 113

Kent, S. M. 1985, ApJS, 59, 115

Kormendy, J. 1977, ApJ, 217, 406

Landolt, A. U. 1983, AJ, 88, 439

Lu, N. Y., Hoffman, G. L., Groff, T., Roos, T., \& Lamphier, C. 1993, ApJS, 88, 383 (Paper I)

Lu, N. Y., Salpeter, E. E., \& Hoffman, G. L. 1994, ApJ, 426, 473

Malmquist, K. G. 1920, Medd. Lunds Astron. Obs., Ser. II, No. 22

McGaugh, S. S. 1996, MNRAS, 280, 337

McGaugh, S. S., Bothun, G. D., \& Schombert, J. M. 1995, AJ, 110, 573

Nilson, P. 1973, Uppsala General Catalogue of Galaxies, Uppsala Astron. Obs. Ann., 6 (UGC)

Pierce, M. J., \& Tully, R. B. 1988, ApJ, 330, 579

Schombert, J. M., Bothun, G. D., Schneider, S. E., McGaugh, S. S. 1992, AJ, 103, 1107

Sprayberry, D., Impey, C. D., \& Irwin, M. J. 1996, ApJ, 463, 535

Szomoru, A., Guhathakurta, P., van Gorkom, J. H., Knapen, J. H., Weinberg, D. H., \& Fruchter, A. S. 1994, AJ, 108, 491

Tully, R. B., \& Fouqué, P. 1985, ApJS, 58, 67

Tully, R. B., \& Verheijen, M. A. W. 1997, ApJ, 484, 145

Tully, R. B., \& Verheijen, M. A. W., Pierce, M. J., Huang, J.-S., Wainscoat, R. J. 1996, AJ, 112, 2471

Valentijn, E. A. 1990, Nature, 346, 153

Valentijn, E. A. 1994, MNRAS, 266, 614

van der Kruit, P. C. 1987, A\&A, 173, 59

Xu, C., \& Buat, V. 1995, A\&A, 293, L65 


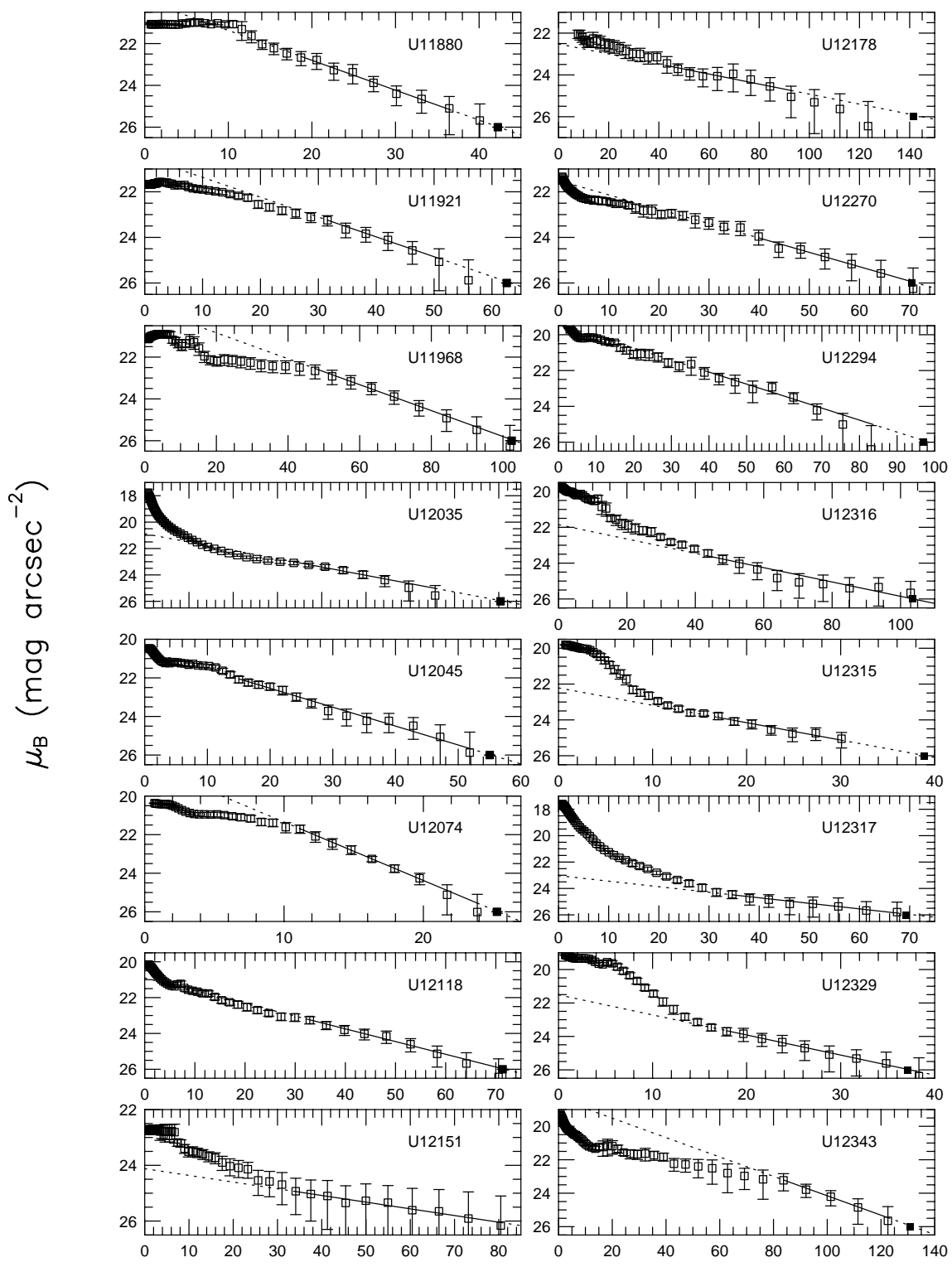

Semi-major axis (arcsec)

Fig. 1.- Plots of the B-band surface brightness as a function of the semi-major axis for the galaxies with photometric data in Table 1. The error bars shown are r.m.s. statistical errors. Open squares are measured isophotes. The solid-to-dashed line is the exponential disk from a fit to the data points covered by the solid line segment. The filled square indicates the B $26 \mathrm{th} \mathrm{mag} \mathrm{arcsec}^{-2}$ isophote determined from the exponential disk fit. 


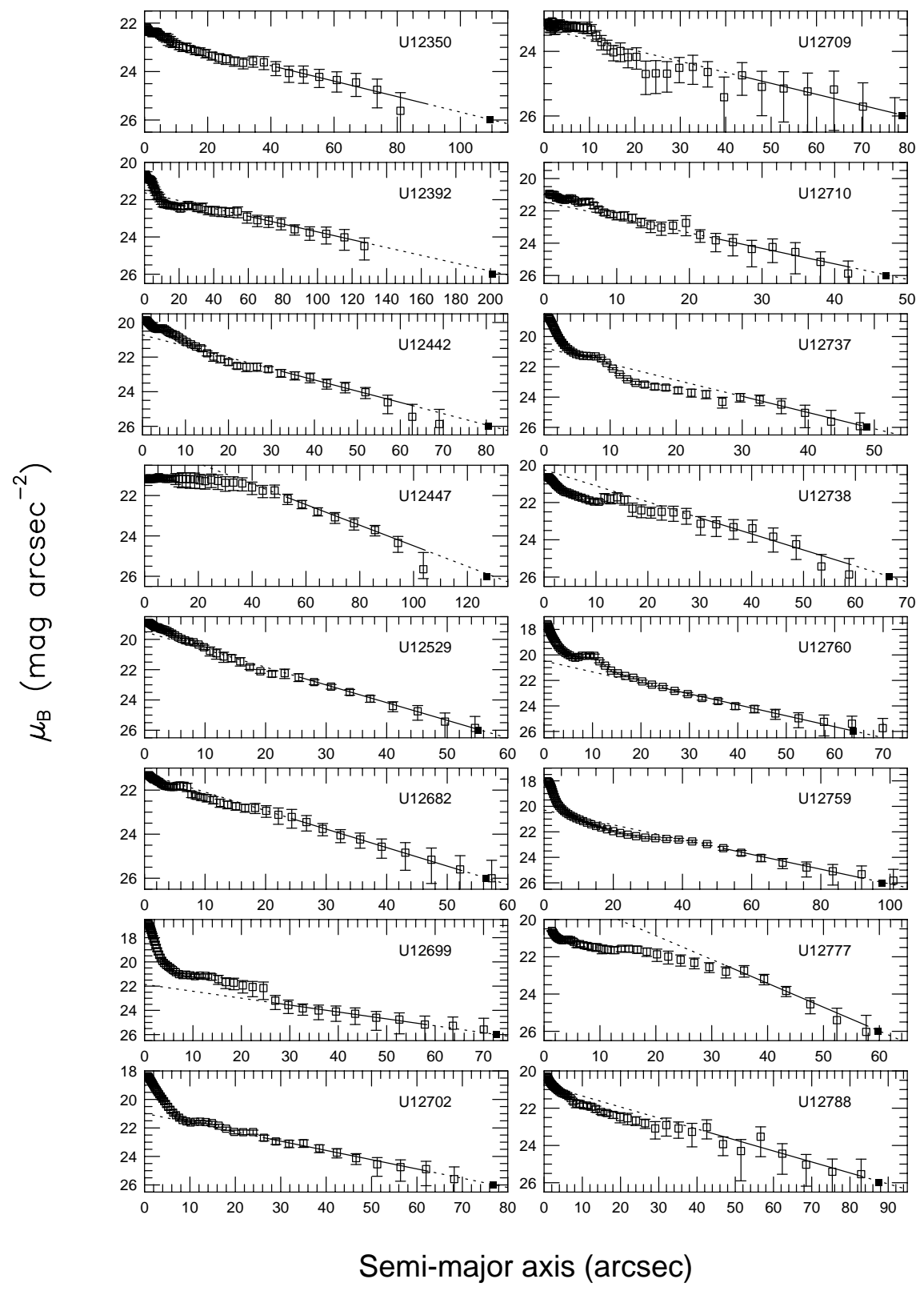

Fig. 1.- Continued. 


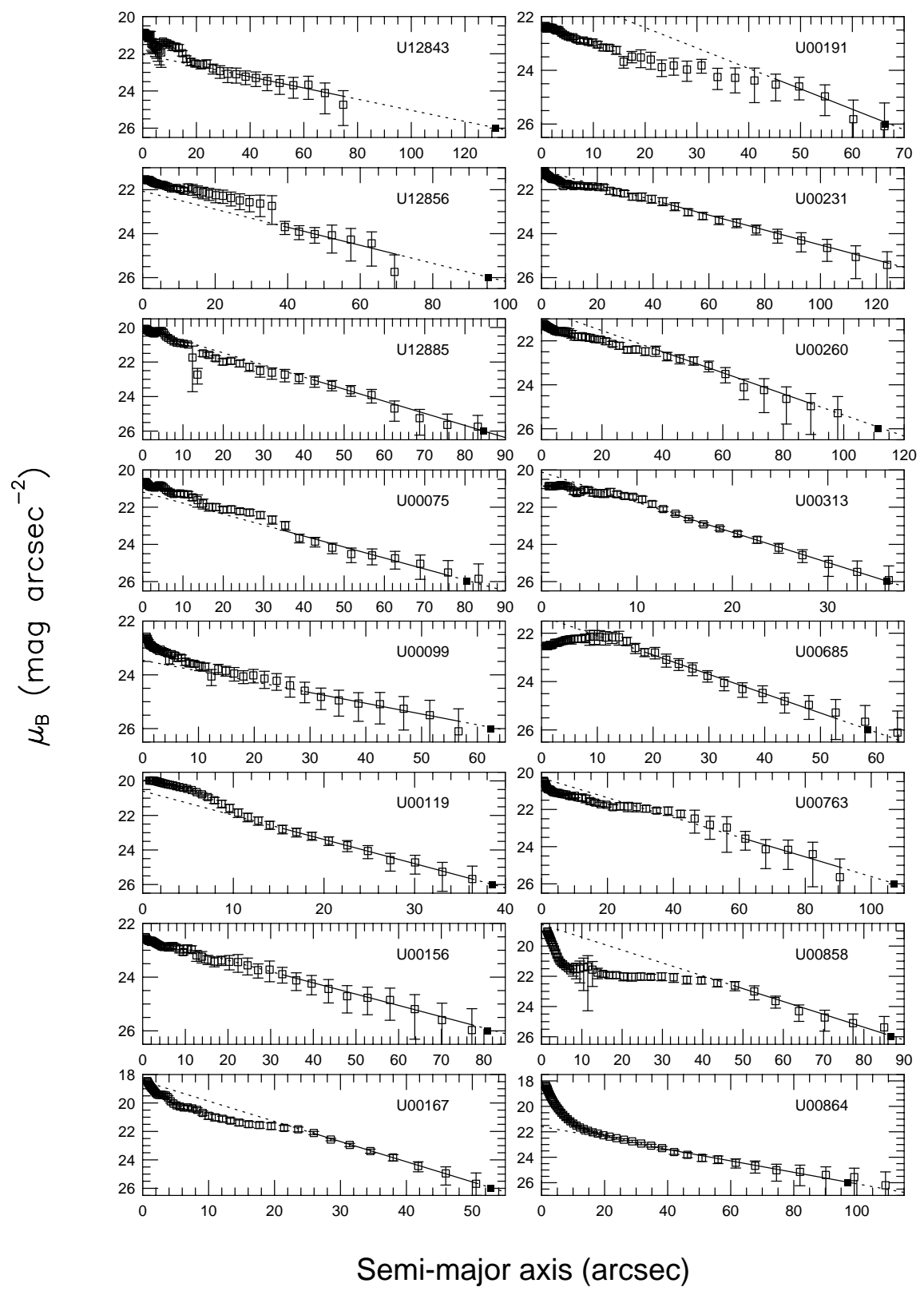

Fig. 1.- Continued. 


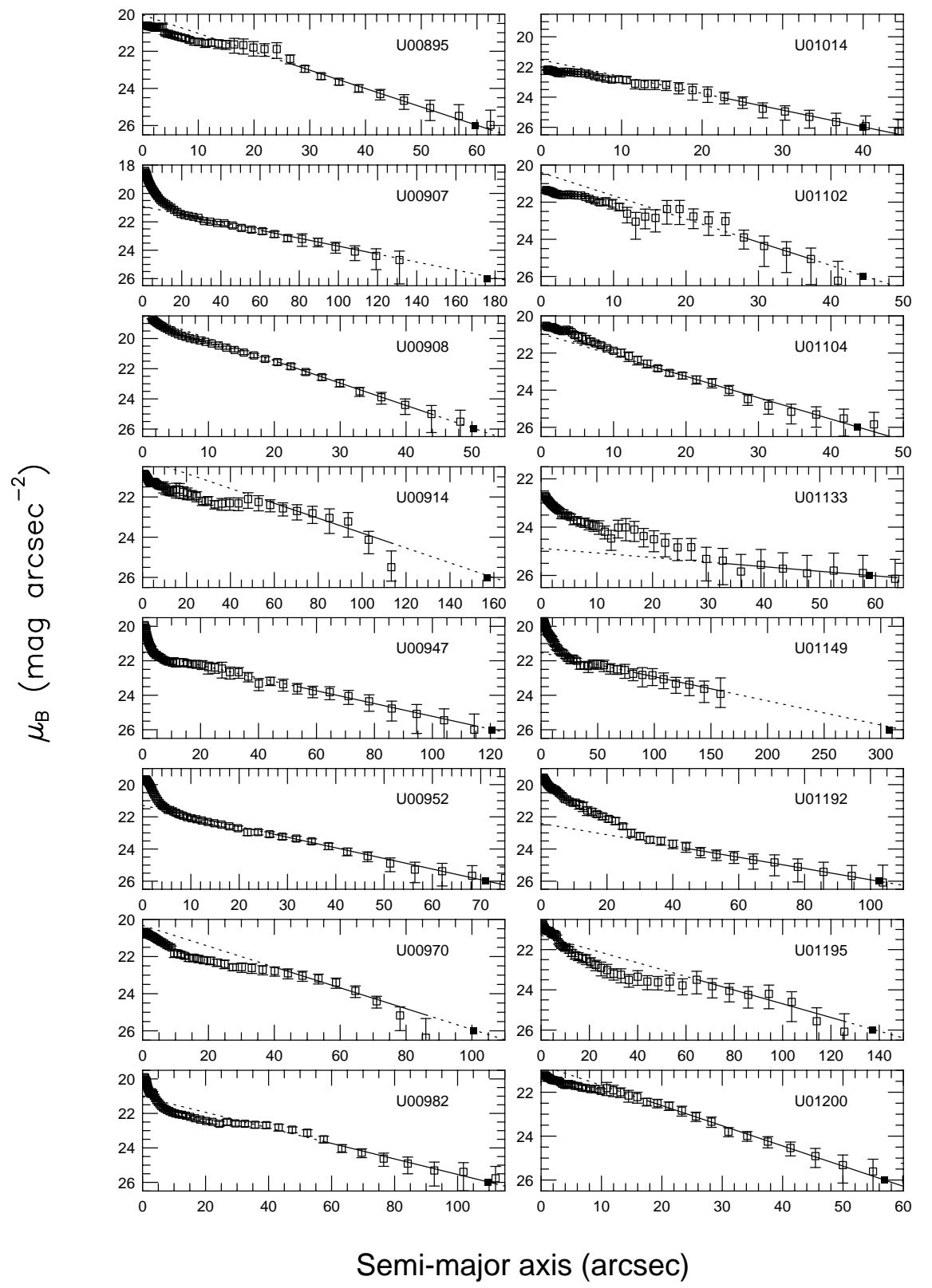

Fig. 1.- Continued. 


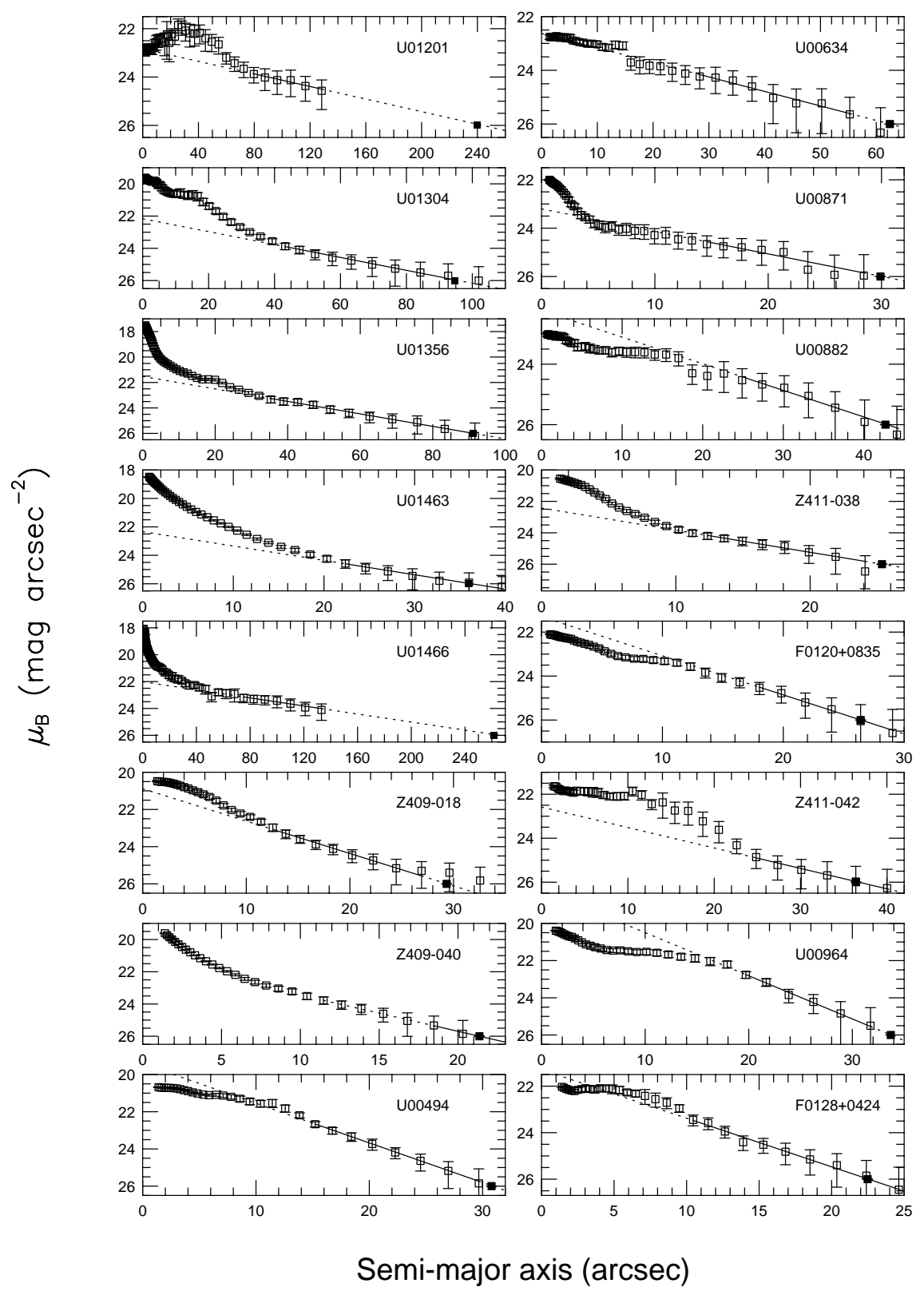

Fig. 1.- Continued. 


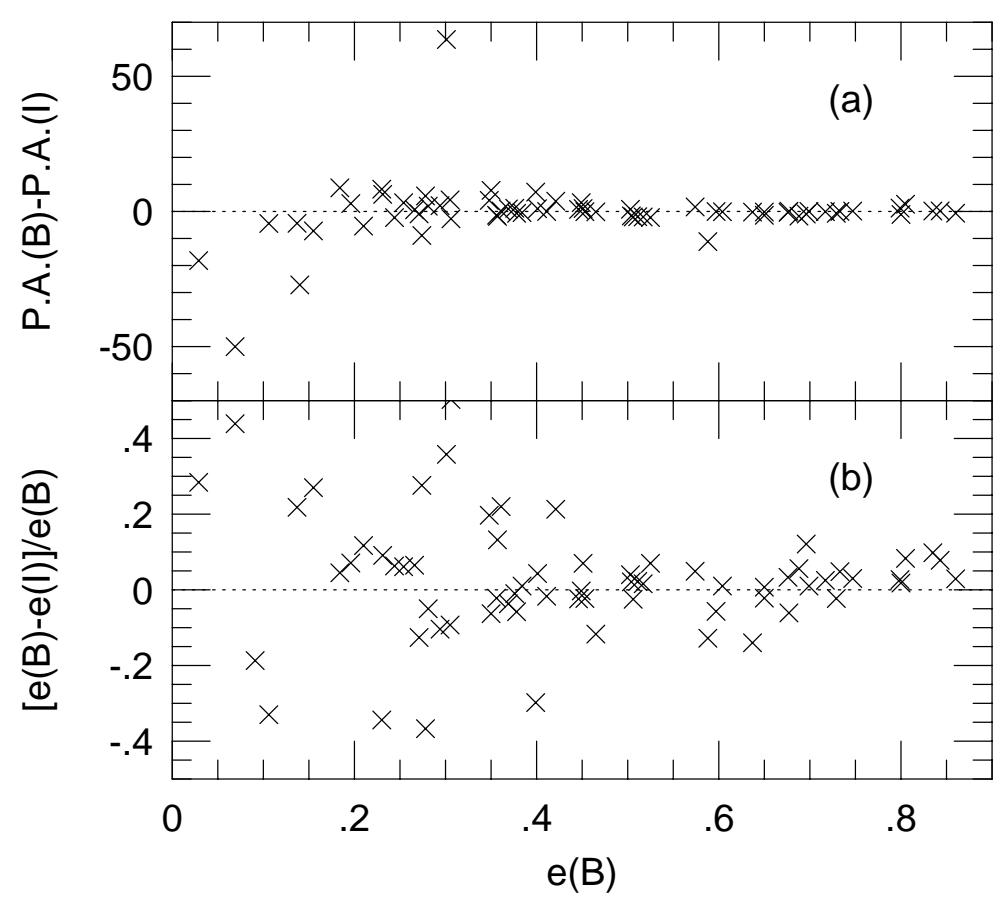

Fig. 2.- Plots as a function of the B-band disk ellipticity of (a) the difference in the mean disk position angle and (b) the relative difference in the mean disk ellipticity between the B-band result in this paper and the I-band result in Lu et al. (1993). Only UGC sample galaxies with available B and I data are shown here. 


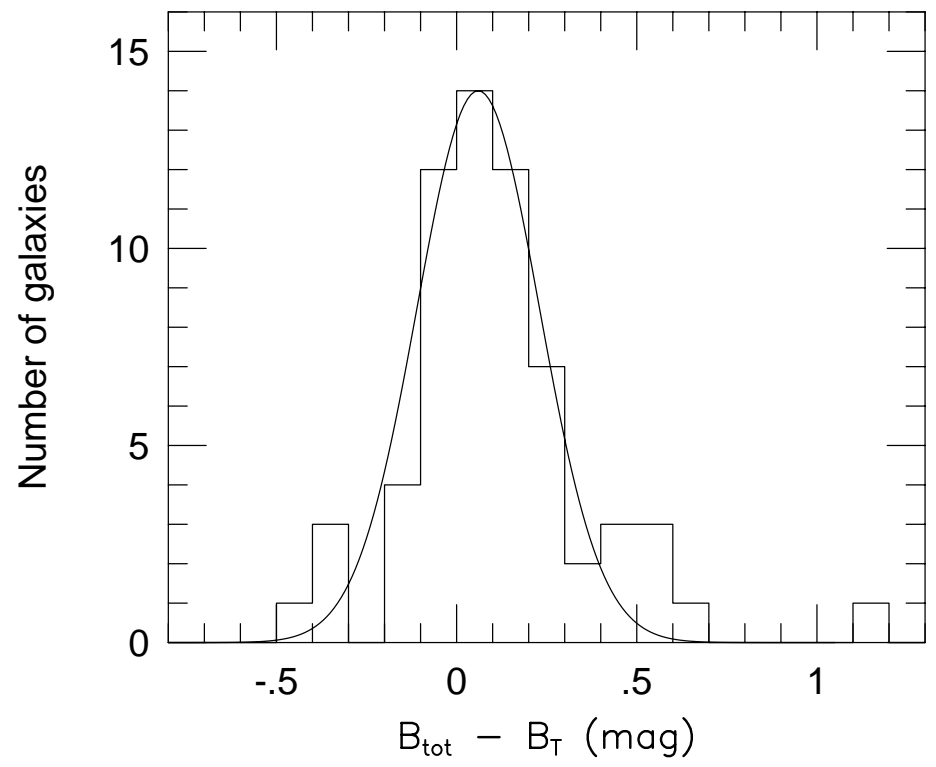

Fig. 3.- Histogram distribution of $\left(B_{\text {tot }}-B_{T}\right)$ for our UGC sample, where $B_{T}$ is taken from the following sources arranged in decreasing preference: $B_{T}$ in RC3, $m_{B}$ in RC3, and the total B magnitude estimated in $\mathrm{Lu}$ et al. (1993). None of the galaxies with notes in Table 1 are used here. The largest magnitude offset in the figure is from UGC 00099, a galaxy of Sd/Sm type without available $B_{T}$ or $m_{B}$ in RC3. The Gaussian curve shown centers at $\left(B_{\text {tot }}-I_{\text {tot }}\right)=0.06$ mag and has a full width at half maximum of 0.4 mag. 


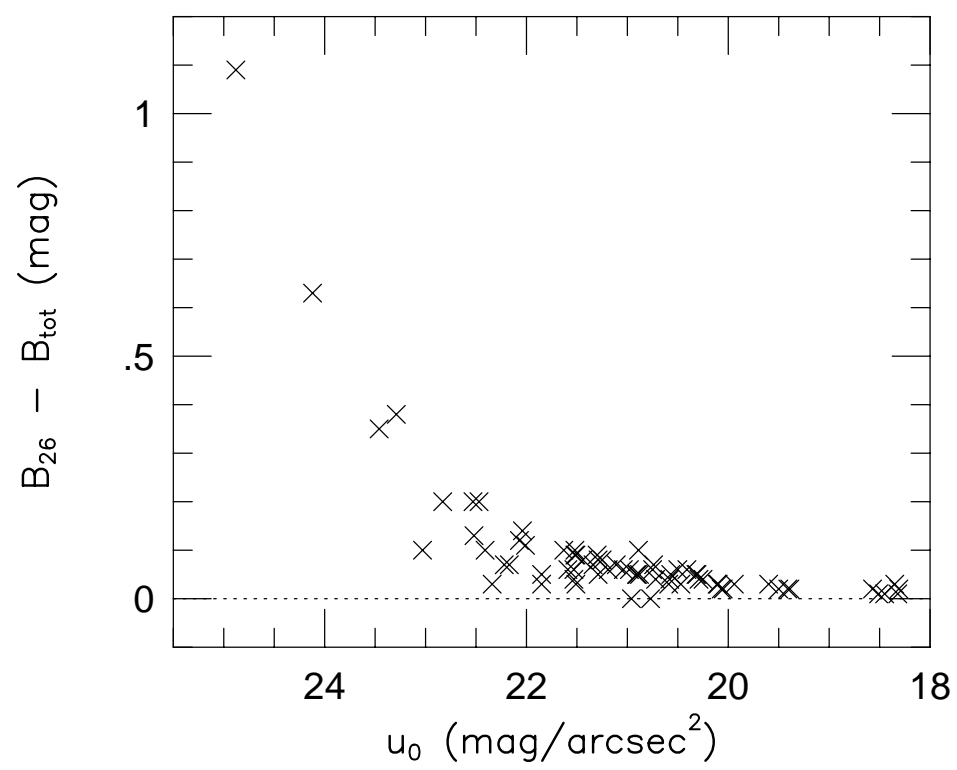

Fig. 4.- Plot of $\left(B_{26}-B_{\text {tot }}\right)$ as a function of the central disk surface brightness for the UGC sample galaxies with available photometry in Table 1 , where $B_{26}$ is the isophotal magnitude at B 26 th- mag arcsec ${ }^{-2}$ and $B_{\text {tot }}$ is the total magnitude. 


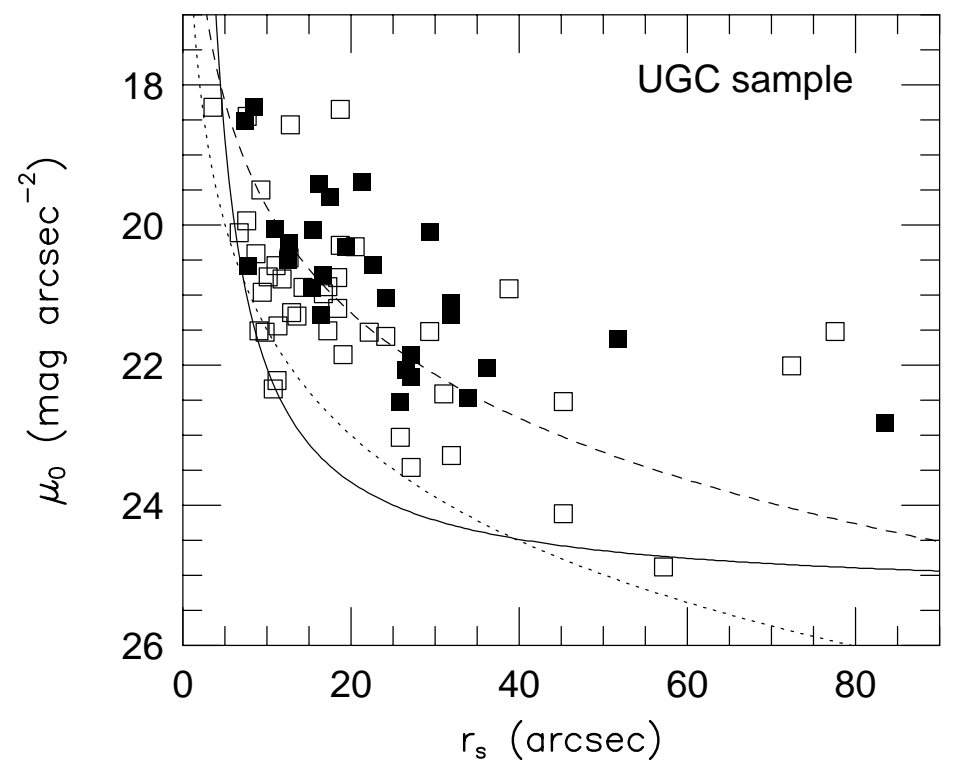

Fig. 5.- Plot of the observed central surface brightness as a function of the observed angular exponential scale length for the disks of the galaxies in the UGC sample. The solid curve represents the diameter selection, eq. (3) in the text; while the dotted and dashed curves sketch respectively the magnitude selection, eq. (5) in the text, for the cases of $e=0$ and $e=0.8$, respectively. The filled (open) squares are galaxies with ellipticities greater (less) than 0.5 . 


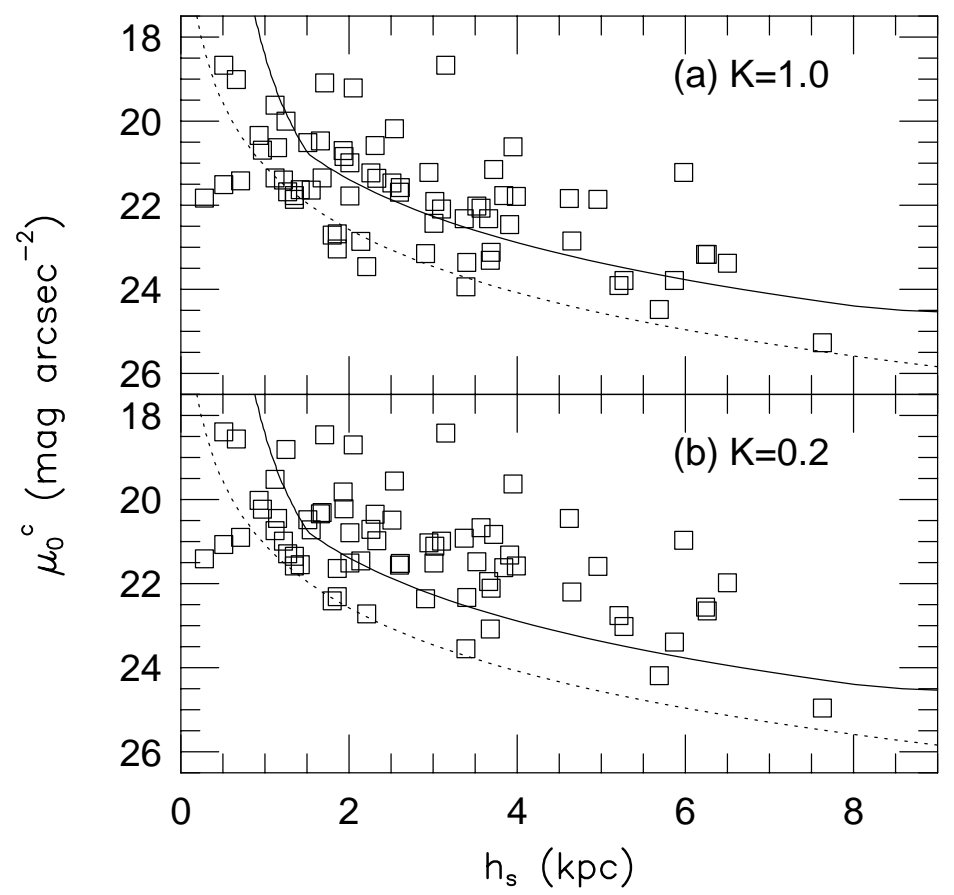

Fig. 6. - Plot of the face-on central surface brightness as a function of the linear disk scale length for the UGC sample galaxies. The dotted curve, given by columns (4) Table 2, indicates the path of a face-on disk galaxy of $M_{B}=-17.5 \mathrm{mag}$ which is roughly detectable up to half of the maximum UGC sample distance. The solid curve, given by column (5) of Table 2, indicates the threshold above which (and to whose right) a face-on galaxy will be detectable up to the maximum sample distance. 


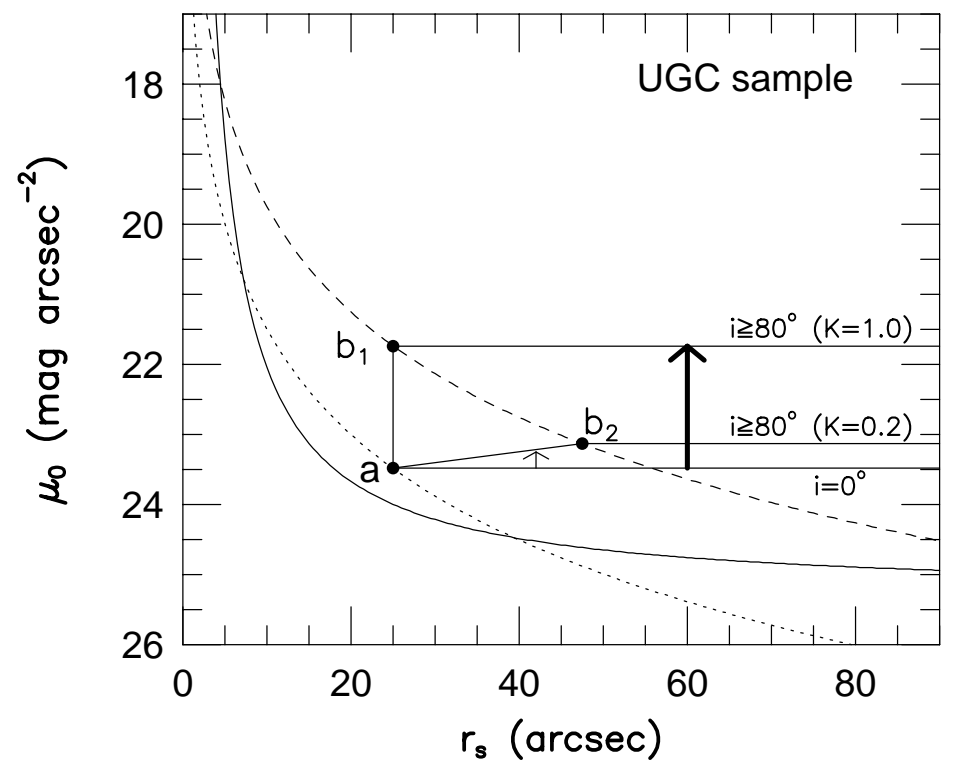

Fig. 7. - A sketch illustrating how a phase space for a disk galaxy is constructed in terms of (1) real space defined by $\mu_{0}$ and $r_{s}$, and (2) a phase space defined by disk inclination angle through eq. (8) in the text. The curves are the same as in Fig. 5. The horizontal lines indicate a disk inclination of $i=0^{\circ}$ and $i \geq 80^{\circ}$, respectively. The thin (thick) arrow sketches how a fully (partially) transparent disk moves as its disk inclination increases at a given distance. 


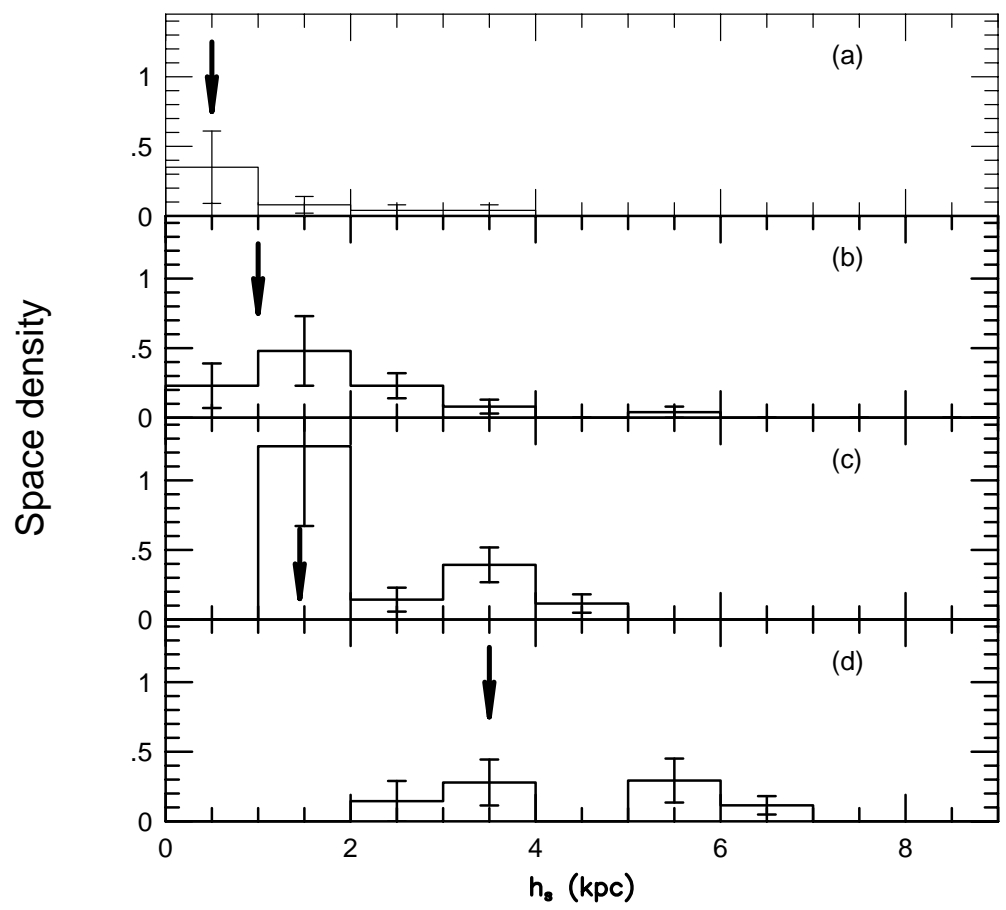

Fig. 8.- Plots of the volume sampling function-adjusted distribution of the disk scale lengths for UGC sample galaxies in that case of transparent disks with $K=1$ : (a) $18<\mu_{0}^{c}<20 \operatorname{mag}^{\operatorname{arcsec}^{-2}}$, (b) $20<\mu_{0}^{c}<21.5 \mathrm{mag} \operatorname{arcsec}^{-2}$, (c) $21.5<\mu_{0}^{c}<23 \mathrm{mag} \mathrm{arcsec}^{-2}$, and (d) $\mu_{0}^{c}>23 \mathrm{mag} \operatorname{arcsec}^{-2}$. The ordinate is in units of number of galaxies per $10^{3} \Omega \mathrm{Mpc}^{3}$, where $\Omega(\approx 0.13 \mathrm{sr})$ is the solid angle covered by our UGC sample on the sky. Only sample galaxies with $M_{B}<-17.5$ mag are used. The error bars are weighted r.m.s. values assuming Poisson statistics. 


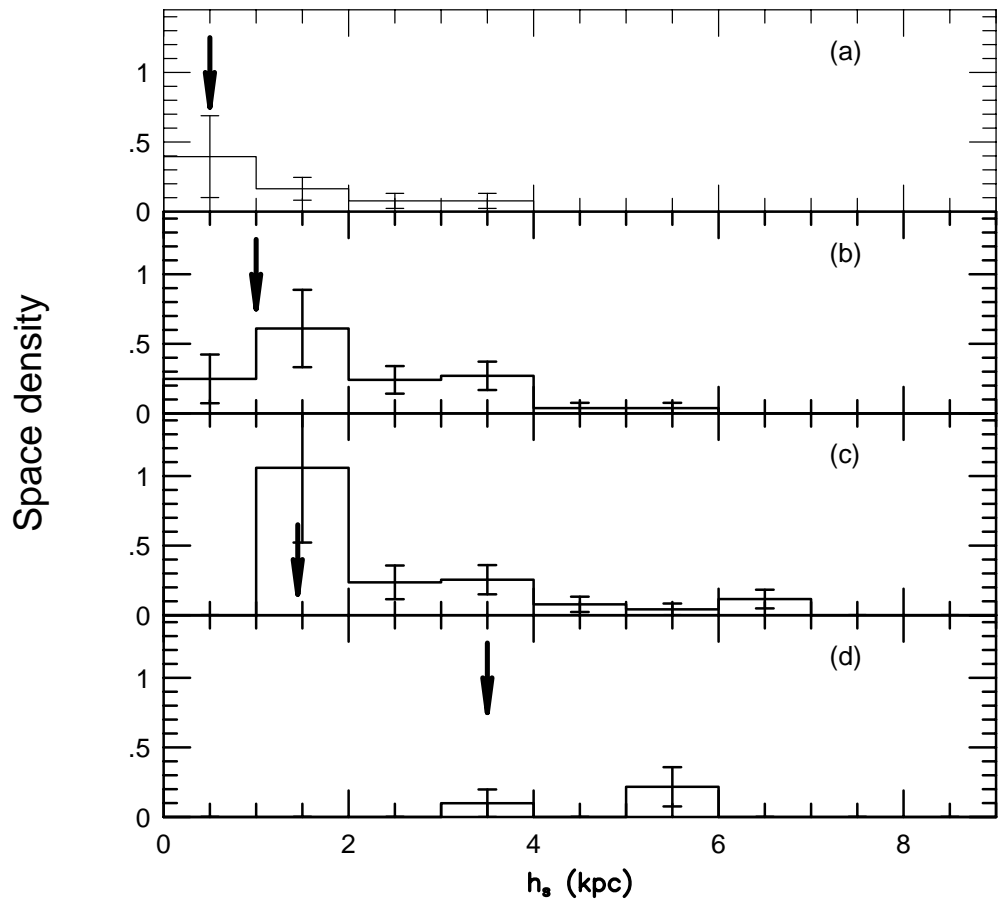

Fig. 9.- The same as in Fig. 8, but for the case of fairly opaque disks with $K=0.2$. 
Table 1. B-band CCD Surface Photometric Results

\begin{tabular}{|c|c|c|c|c|c|c|c|c|c|c|c|c|c|c|c|}
\hline $\begin{array}{l}\text { UGC } \\
(1)\end{array}$ & $\begin{array}{c}\mathrm{NGC} / \mathrm{IC} \\
(2)\end{array}$ & $\begin{array}{l}\text { Dist. } \\
(3)\end{array}$ & $\begin{array}{l}r_{1} \\
(4)\end{array}$ & $\begin{array}{l}r_{2} \\
(5)\end{array}$ & $\begin{array}{c}e \\
(6)\end{array}$ & $\begin{array}{c}i \\
(7)\end{array}$ & $\begin{array}{l}\text { P.A. } \\
(8)\end{array}$ & $\begin{array}{c}B_{26} \\
(9)\end{array}$ & $\begin{array}{l}D_{26} \\
(10)\end{array}$ & $\begin{array}{c}r_{s} \\
(11)\end{array}$ & $\begin{array}{c}h_{s} \\
(12)\end{array}$ & $\begin{array}{c}\mu_{0} \\
(13)\end{array}$ & $\begin{array}{l}B_{t o t} \\
(14)\end{array}$ & $\begin{array}{l}M_{B} \\
(15)\end{array}$ & $\begin{array}{c}(B-I) \\
(16)\end{array}$ \\
\hline I. The UGC & Sample: & & & & & & & & & & & & & & \\
\hline U11880 & I1420 & 25.33 & 18.7 & 36.4 & 0.306 & 47 & 112 & 14.20 & 1.41 & 7.6 & 0.93 & 19.94 & 14.17 & -17.85 & 1.54 \\
\hline U11921 & $\ldots$ & 25.63 & 31.6 & 50.9 & 0.650 & 72 & 124 & 14.62 & 2.09 & 12.5 & 1.55 & 20.50 & 14.56 & -17.48 & 1.37 \\
\hline U11968 & N7241 & 22.71 & 47.6 & 101.9 & 0.637 & 71 & 21 & 13.02 & 3.41 & 17.5 & 1.93 & 19.60 & 12.99 & -18.79 & 1.94 \\
\hline U12035 & N7280 & 27.91 & 33.7 & 65.6 & 0.356 & 51 & 74 & 13.09 & 2.68 & 17.2 & 2.33 & 20.88 & 13.04 & -19.19 & 2.10 \\
\hline U12045 & N7290 & 42.04 & 15.0 & 51.8 & 0.449 & 58 & 161 & 14.03 & 1.83 & 11.1 & 2.26 & 20.58 & 13.99 & -19.13 & 1.58 \\
\hline U12074 & $\ldots$ & 29.42 & 11.1 & 23.9 & 0.281 & 45 & 149 & 14.71 & 0.84 & 3.6 & 0.51 & 18.32 & 14.70 & -17.64 & 2.04 \\
\hline U12118 & N7328 & 40.67 & 32.9 & 70.5 & 0.604 & 69 & 86 & 13.95 & 2.38 & 15.3 & 3.02 & 20.90 & 13.90 & -19.15 & 1.96 \\
\hline U12151 & $\ldots$ & 25.94 & 34.1 & 80.5 & 0.278 & 44 & 0 & 14.80 & 2.61 & 45.2 & 5.69 & 24.12 & 14.17 & -17.90 & 1.36 \\
\hline $\mathrm{U} 12178^{\mathrm{a}}$ & $\ldots$ & 28.54 & 43.3 & 92.7 & 0.453 & 58 & 9 & 13.06 & 4.72 & 45.2 & 6.26 & 22.52 & 12.87 & -19.75 & 1.24 \\
\hline U12270 & N7437 & 31.28 & 39.9 & 70.7 & 0.069 & 21 & 43 & 13.57 & 2.35 & 17.2 & 2.61 & 21.51 & 13.48 & -19.00 & 1.48 \\
\hline U12294 & N7448 & 32.37 & 38.8 & 83.1 & 0.511 & 62 & 171 & 12.24 & 3.24 & 16.2 & 2.54 & 19.41 & 12.22 & -20.33 & 1.48 \\
\hline U12316 & N7463 & 28.02 & 43.7 & 113.2 & 0.688 & 75 & 93 & 13.37 & 3.45 & 27.1 & 3.69 & 21.85 & 13.34 & -18.90 & 1.42 \\
\hline U12315 & N7464 & 34.26 & 17.0 & 30.1 & 0.348 & 50 & 154 & 14.71 & 1.30 & 11.2 & 1.86 & 22.22 & 14.64 & -18.03 & 1.01 \\
\hline U12317 & N7465 & 29.35 & 34.6 & 67.5 & 0.230 & 40 & 165 & 13.30 & 2.31 & 25.9 & 3.68 & 23.03 & 13.20 & -19.14 & 1.72 \\
\hline U12329 & N7468 & 30.89 & 17.9 & 38.3 & 0.274 & 44 & 19 & 14.19 & 1.24 & 9.1 & 1.35 & 21.51 & 14.16 & -18.29 & 1.30 \\
\hline U12343 & N7479 & 34.71 & 83.7 & 122.6 & 0.254 & 42 & 39 & 11.81 & 4.36 & 18.7 & 3.15 & 18.35 & 11.78 & -20.92 & 1.99 \\
\hline U12350 & $\ldots$ & 31.66 & 41.6 & 89.1 & 0.733 & 79 & 95 & 14.48 & 3.64 & 33.9 & 5.21 & 22.47 & 14.28 & -18.22 & 1.52 \\
\hline U12392 & N7497 & 25.93 & 59.3 & 127.1 & 0.800 & 90 & 42 & 12.98 & 6.71 & 51.7 & 6.50 & 21.63 & 12.88 & -19.19 & 1.87 \\
\hline U12442 & N7537 & 38.25 & 32.2 & 62.8 & 0.717 & 78 & 77 & 13.85 & 2.68 & 16.7 & 3.10 & 20.72 & 13.81 & -19.10 & 1.81 \\
\hline U12447 & N7541 & 38.23 & 53.2 & 103.6 & 0.676 & 74 & 100 & 12.37 & 4.24 & 21.3 & 3.95 & 19.39 & 12.35 & -20.56 & 2.06 \\
\hline $\mathrm{U} 12529$ & $\begin{array}{c}\text { N7625 } \\
\ldots\end{array}$ & 24.83 & 25.5 & 54.6 & 0.106 & 27 & 32 & 12.86 & 1.84 & $\begin{array}{l}9.3 \\
\ldots\end{array}$ & 1.12 & 19.50 & 12.84 & -19.13 & 2.02 \\
\hline U12682 & $\ldots$ & 21.57 & 24.3 & 52.1 & 0.378 & 53 & 43 & 14.35 & 1.88 & 12.9 & 1.35 & 21.25 & 14.27 & -17.40 & 1.27 \\
\hline U12699 & N7714 & 39.59 & 27.0 & 57.9 & 0.350 & 50 & 37 & 13.04 & 2.42 & 19.1 & 2.66 & 21.85 & 12.99 & -20.00 & 1.69 \\
\hline U12702 & N7716 & 36.48 & 26.3 & 62.0 & 0.196 & 37 & 45 & 13.03 & 2.56 & 16.7 & 2.95 & 20.98 & 12.97 & -19.84 & 1.85 \\
\hline U12709 & $\ldots$ & 37.88 & 43.6 & 77.2 & 0.369 & 52 & 144 & 14.69 & 2.63 & 31.9 & 5.87 & 23.29 & 14.31 & -18.58 & 1.44 \\
\hline U12710 & $\ldots$ & 36.58 & 23.6 & 41.9 & 0.266 & 43 & 162 & 14.48 & 1.57 & 11.3 & 2.01 & 21.44 & 14.40 & -18.42 & 1.20 \\
\hline U12737 & N7731 & 40.81 & 29.7 & 47.8 & 0.210 & 38 & 91 & 14.26 & 1.63 & 10.1 & 2.01 & 20.74 & 14.19 & -18.86 & 1.88 \\
\hline U12738 & N7732 & 41.09 & 30.2 & 58.8 & 0.677 & 74 & 89 & 14.33 & 2.22 & 12.6 & 2.51 & 20.25 & 14.29 & -18.78 & 1.51 \\
\hline U12760 & N7742 & 24.65 & 24.5 & 63.6 & 0.029 & 14 & 50 & 12.50 & 2.13 & 12.6 & 1.51 & 20.47 & 12.47 & -19.49 & 1.87 \\
\hline U12759 & N7743 & 25.40 & 51.8 & 91.8 & 0.231 & 40 & 75 & 12.42 & 3.25 & 18.7 & 2.31 & 20.29 & 12.38 & -19.64 & 2.16 \\
\hline U12777 & N7750 & 41.47 & 32.5 & 57.6 & 0.517 & 63 & 173 & 13.56 & 1.99 & 8.5 & 1.71 & 18.31 & 13.54 & -19.55 & 1.77 \\
\hline U12788 & N7757 & 41.71 & 42.6 & 82.9 & 0.305 & 47 & 123 & 13.09 & 2.92 & 18.4 & 3.72 & 20.75 & 13.03 & -20.07 & 1.26 \\
\hline $\mathrm{U} 12843^{\mathrm{c}}$ & $\ldots$ & 26.53 & 34.8 & 74.7 & 0.525 & 63 & 24 & 13.14 & 4.38 & 36.2 & 4.65 & 22.04 & 13.00 & -19.12 & 1.12 \\
\hline U12856 & $\cdots$ & 26.45 & 39.2 & 69.5 & 0.696 & 76 & 15 & 14.15 & 3.17 & 26.5 & 3.40 & 22.07 & 14.03 & -18.08 & 0.96 \\
\hline U12885 & N7800 & 25.84 & 42.7 & 91.5 & 0.506 & 62 & 45 & 13.28 & 2.82 & 15.5 & 1.94 & 20.07 & 13.26 & -18.80 & 1.36 \\
\hline U00008 ${ }^{\mathrm{d}}$ & N7814 & $\ldots$ & $\ldots$ & $\ldots$ & $\ldots$ & $\ldots$ & $\ldots$ & $\ldots$ & $\ldots$ & $\ldots$ & $\ldots$ & $\ldots$ & $\ldots$ & $\ldots$ & $\ldots$ \\
\hline U00075 & N0014 & 14.19 & 35.4 & 75.8 & 0.361 & 51 & 28 & 13.28 & 2.68 & 18.4 & 1.27 & 21.19 & 13.22 & -17.54 & 1.69 \\
\hline U00099 & $\ldots$ & 25.77 & 29.1 & 56.6 & 0.357 & 51 & 154 & 15.00 & 2.08 & 27.1 & 3.39 & 23.46 & 14.65 & -17.41 & 1.24 \\
\hline U00119 & $\ldots$ & 29.66 & 15.4 & 36.4 & 0.504 & 62 & 81 & 14.62 & 1.29 & 7.8 & 1.12 & 20.59 & 14.59 & -17.77 & 1.34 \\
\hline U00156 & $\ldots$ & 17.60 & 32.7 & 77.2 & 0.574 & 67 & 0 & 14.62 & 2.69 & 25.9 & 2.21 & 22.53 & 14.42 & -16.81 & 1.58 \\
\hline U00167 & N0063 & 17.87 & 25.9 & 50.6 & 0.401 & 54 & 104 & 13.17 & 1.76 & 7.6 & 0.66 & 18.45 & 13.16 & -18.10 & 1.89 \\
\hline U00191 & $\ldots$ & 17.61 & 45.2 & 66.2 & 0.376 & 52 & 154 & 14.42 & 2.21 & 14.3 & 1.22 & 20.89 & 14.32 & -16.91 & 1.60 \\
\hline U00231 & N0100 & 13.82 & 43.4 & 123.9 & 0.860 & 90 & 55 & 13.84 & 4.66 & 31.9 & 2.14 & 21.11 & 13.77 & -16.93 & 1.71 \\
\hline U00260 & $\ldots$ & 30.76 & 37.8 & 89.1 & 0.843 & 90 & 22 & 14.03 & 3.71 & 22.6 & 3.37 & 20.57 & 13.98 & -18.46 & 1.57 \\
\hline U00313 & $\ldots$ & 29.84 & 14.0 & 36.4 & 0.411 & 55 & 13 & 14.62 & 1.21 & 6.7 & 0.97 & 20.11 & 14.59 & -17.78 & 1.61 \\
\hline $\mathrm{U} 00668^{\mathrm{b}}$ & I1613 & $\ldots$ & $\ldots$ & $\ldots$ & $\ldots$ & $\ldots$ & $\ldots$ & $\ldots$ & $\ldots$ & $\ldots$ & $\ldots$ & $\ldots$ & $\ldots$ & $\ldots$ & $\ldots$ \\
\hline U00685 & $\cdots$ & 4.27 & 18.5 & 52.7 & 0.384 & 53 & 116 & 14.32 & 1.95 & 13.6 & 0.28 & 21.30 & 14.23 & -13.92 & 1.51 \\
\hline $\mathrm{U} 00711^{\mathrm{d}}$ & $\cdots$ & $\cdots$ & $\cdots$ & $\cdots$ & & 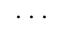 & - & & & 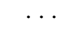 & 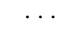 & & & & \\
\hline U00763 & N0428 & 16.76 & 61.8 & 90.5 & 0.140 & 31 & 73 & 12.17 & 3.57 & 20.5 & 1.66 & 20.31 & 12.12 & -19.00 & 1.43 \\
\hline U00858 & N0470 & 33.04 & 48.0 & 85.0 & 0.446 & 58 & 151 & 12.69 & 2.89 & 12.8 & 2.05 & 18.57 & 12.67 & -19.93 & 1.98 \\
\hline
\end{tabular}


Table 1 - Continued

\begin{tabular}{|c|c|c|c|c|c|c|c|c|c|c|c|c|c|c|c|}
\hline $\begin{array}{l}\text { UGC } \\
(1)\end{array}$ & $\begin{array}{c}\mathrm{NGC} / \mathrm{IC} \\
(2)\end{array}$ & $\begin{array}{l}\text { Dist. } \\
(3)\end{array}$ & $\begin{array}{l}r_{1} \\
(4)\end{array}$ & $\begin{array}{l}r_{2} \\
(5)\end{array}$ & $\begin{array}{c}e \\
(6)\end{array}$ & $\begin{array}{c}i \\
(7)\end{array}$ & $\begin{array}{l}\text { P.A. } \\
(8)\end{array}$ & $\begin{array}{c}B_{26} \\
(9)\end{array}$ & $\begin{array}{l}D_{26} \\
(10)\end{array}$ & $\begin{array}{l}r_{s} \\
(11)\end{array}$ & $\begin{array}{c}h_{s} \\
(12)\end{array}$ & $\begin{array}{c}\mu_{0} \\
(13)\end{array}$ & $\begin{array}{l}B_{t o t} \\
(14)\end{array}$ & $\begin{array}{l}M_{B} \\
(15)\end{array}$ & $\begin{array}{c}(B-I) \\
\quad(16)\end{array}$ \\
\hline U00859 ${ }^{\mathrm{e}}$ & N0473 & $\ldots$ & $\ldots$ & $\ldots$ & $\ldots$ & $\ldots$ & $\ldots$ & $\ldots$ & $\ldots$ & $\ldots$ & $\ldots$ & $\ldots$ & $\ldots$ & $\ldots$ & $\ldots$ \\
\hline U00864 & N0474 & 32.85 & 34.8 & 99.2 & 0.155 & 33 & 10 & 12.62 & 3.24 & 24.1 & 3.84 & 21.59 & 12.56 & -20.02 & 2.35 \\
\hline U00895 & N0485 & 31.56 & 26.5 & 62.4 & 0.699 & 76 & 4 & 14.24 & 1.99 & 11.0 & 1.68 & 20.05 & 14.22 & -18.28 & 1.91 \\
\hline U00907 & N0488 & 31.80 & 61.2 & 119.2 & 0.244 & 41 & 4 & 11.28 & 5.86 & 38.8 & 5.98 & 20.91 & 11.23 & -21.28 & 2.18 \\
\hline U00908 & N0489 & 35.09 & 20.5 & 43.9 & 0.747 & 80 & 120 & 13.73 & 1.68 & 7.3 & 1.25 & 18.51 & 13.72 & -19.01 & 2.30 \\
\hline U00914 & N0493 & 32.46 & 58.1 & 113.3 & 0.805 & 90 & 61 & 12.82 & 5.23 & 29.4 & 4.62 & 20.10 & 12.79 & -19.77 & 1.27 \\
\hline U00947 & N0514 & 34.84 & 53.4 & 114.4 & 0.271 & 44 & 102 & 12.64 & 4.01 & 29.4 & 4.96 & 21.52 & 12.54 & -20.17 & 1.71 \\
\hline U00952 & N0518 & 37.73 & 26.3 & 75.0 & 0.650 & 72 & 97 & 14.37 & 2.37 & 16.5 & 3.01 & 21.29 & 14.32 & -18.56 & 2.48 \\
\hline U00966 ${ }^{\mathrm{f}}$ & N0520 & ... & $\ldots$ & $\ldots$ & $\ldots$ & $\ldots$ & $\ldots$ & $\ldots$ & $\ldots$ & $\ldots$ & $\ldots$ & $\ldots$ & $\ldots$ & $\ldots$ & $\ldots$ \\
\hline U00970 & N0522 & 37.97 & 40.1 & 86.0 & 0.835 & 90 & 33 & 14.14 & 3.35 & 19.4 & 3.57 & 20.32 & 14.09 & -18.81 & 2.51 \\
\hline U00982 & N0532 & 33.41 & 57.5 & 112.0 & 0.729 & 79 & 29 & 13.66 & 3.65 & 24.1 & 3.91 & 21.04 & 13.60 & -19.02 & 2.44 \\
\hline U01014 & $\ldots$ & 29.94 & 22.8 & 44.3 & 0.091 & 25 & 174 & 14.83 & 1.33 & 9.8 & 1.42 & 21.53 & 14.79 & -17.59 & 1.00 \\
\hline U01102 & $\ldots$ & 27.42 & 28.0 & 37.2 & 0.184 & 36 & 0 & 14.43 & 1.48 & 8.7 & 1.15 & 20.41 & 14.37 & -17.82 & 0.67 \\
\hline U01104 & $\ldots$ & 11.15 & 13.3 & 50.5 & 0.399 & 54 & 6 & 14.49 & 1.45 & 9.4 & 0.51 & 20.96 & 14.49 & -15.75 & 2.31 \\
\hline U01133 & $\ldots$ & 27.55 & 32.6 & 63.5 & 0.301 & 46 & 177 & 15.52 & 1.96 & 57.2 & 7.63 & 24.88 & 14.43 & -17.77 & $\ldots$ \\
\hline U01149g & N0628 & 10.61 & 81.3 & 158.5 & 0.217 & 39 & 148 & 10.47 & 10.26 & 77.6 & 3.99 & 21.52 & 10.38 & -19.75 & 1.55 \\
\hline U01192 & N0658 & 41.49 & 44.0 & 103.8 & 0.503 & 62 & 28 & 13.27 & 3.42 & 31.0 & 6.24 & 22.41 & 13.17 & -19.92 & 1.71 \\
\hline U01195 & $\ldots$ & 12.03 & 64.4 & 125.5 & 0.799 & 88 & 48 & 13.70 & 4.57 & 31.9 & 1.86 & 21.29 & 13.62 & -16.78 & 1.36 \\
\hline U01200 & $\cdots$ & 12.45 & 17.5 & 66.5 & 0.451 & 58 & 168 & 14.13 & 1.90 & 11.8 & 0.71 & 20.77 & 14.13 & -16.35 & 1.48 \\
\hline U01201 & N0660 & 13.02 & 79.7 & 128.4 & 0.588 & 68 & 23 & 12.16 & 8.00 & 83.5 & 5.27 & 22.83 & 11.96 & -18.61 & 2.21 \\
\hline $\mathrm{U} 01270^{\mathrm{e}}$ & N0676 & $\ldots$ & $\ldots$ & $\ldots$ & $\ldots$ & .. & $\ldots$ & $\ldots$ & $\ldots$ & $\ldots$ & $\ldots$ & $\ldots$ & $\ldots$ & $\ldots$ & $\ldots$ \\
\hline U01304 & N0693 & 22.11 & 43.2 & 92.7 & 0.597 & 69 & 104 & 13.33 & 3.15 & 27.1 & 2.91 & 22.17 & 13.26 & -18.46 & 2.24 \\
\hline U01356 & N0718 & 24.23 & 35.3 & 91.6 & 0.137 & 30 & 23 & 12.63 & 3.04 & 22.2 & 2.60 & 21.53 & 12.57 & -19.35 & $\ldots$ \\
\hline U01463 & N0770 & 34.49 & 22.4 & 39.6 & 0.294 & 46 & 10 & 14.45 & 1.20 & 10.8 & 1.80 & 22.34 & 14.42 & -18.27 & $\ldots$ \\
\hline U01466 ${ }^{\mathrm{h}}$ & N0772 & 34.71 & 75.1 & 133.1 & 0.421 & 56 & 124 & 11.25 & 8.73 & 72.4 & 12.18 & 22.01 & 11.14 & -21.56 & 1.76 \\
\hline II. Fainter & Galaxies: & & & & & & & & & & & & & & \\
\hline Z409-018 & $\ldots$ & 19.93 & 12.6 & 26.9 & 0.479 & 60 & 6 & 15.40 & 0.98 & 6.2 & 0.60 & 20.89 & 15.36 & -16.14 & 1.51 \\
\hline Z409-040 & $\ldots$ & 9.47 & 11.5 & 18.5 & 0.400 & 54 & 135 & 15.67 & 0.71 & 4.8 & 0.22 & 21.19 & 15.63 & -14.25 & 2.33 \\
\hline U00494 & $\ldots$ & 27.93 & 15.2 & 29.7 & 0.585 & 68 & 95 & 15.21 & 1.03 & 5.1 & 0.69 & 19.42 & 15.19 & -17.04 & 1.40 \\
\hline U00634 & $\ldots$ & 31.38 & 28.4 & 55.2 & 0.567 & 66 & 34 & 15.25 & 2.08 & 20.1 & 3.06 & 22.63 & 15.03 & -17.45 & 1.15 \\
\hline U00871 & $\cdots$ & 30.36 & 14.6 & 28.4 & 0.271 & 44 & 133 & 16.40 & 1.00 & 11.7 & 1.72 & 23.20 & 16.10 & -16.31 & 1.61 \\
\hline U00882 & $\ldots$ & 32.67 & 24.9 & 44.1 & 0.404 & 55 & 82 & 15.72 & 1.42 & 12.3 & 1.96 & 22.23 & 15.56 & -17.01 & 1.50 \\
\hline Z411-038 & $\ldots$ & 36.84 & 12.4 & 24.1 & 0.229 & 40 & 11 & 15.89 & 0.85 & 7.7 & 1.37 & 22.42 & 15.78 & -17.05 & 1.54 \\
\hline $\mathrm{F} 0120+0835^{\mathrm{i}}$ & $\ldots$ & 31.26 & 18.0 & 29.0 & 0.015 & 10 & 149 & 15.83 & 0.88 & 6.1 & 0.92 & 21.30 & 15.78 & -16.70 & 1.82 \\
\hline Z411-042 & $\ldots$ & 37.62 & 24.9 & 40.0 & 0.466 & 59 & 153 & 15.33 & 1.21 & 11.7 & 2.13 & 22.58 & 15.27 & -17.61 & 0.74 \\
\hline U00964 & $\ldots$ & 38.09 & 19.7 & 31.8 & 0.864 & 80 & 90 & 15.40 & 1.12 & 4.7 & 0.86 & 18.17 & 15.38 & -17.52 & 1.06 \\
\hline F0128+0424 & $\ldots$ & 28.68 & 10.4 & 24.6 & 0.336 & 49 & 133 & 16.27 & 0.75 & 5.2 & 0.72 & 21.29 & 16.24 & -16.05 & 1.46 \\
\hline
\end{tabular}

${ }^{\text {a }}$ Photometry may be affected by bright stars nearby.

${ }^{\mathrm{b}}$ Local Group dwarf galaxies. Not observed.

${ }^{c}$ The exposure time is only $60 \mathrm{sec}$ due to a bright foreground star.

${ }^{\mathrm{d}}$ Extremely edge-on galaxies. No surface photometry.

No data.

${ }^{\mathrm{f}}$ An interacting galaxy pair. No surface photometry.

${ }^{\mathrm{g}}$ The galaxy occupies most of the CCD field and the sky subtraction may be less accurate.

${ }^{\mathrm{h}}$ The disk fit is performed over a range of radii where spiral arms of the galaxy are prominent.

${ }^{\mathrm{i}}=\mathrm{MCG}+01-04-042$. 
Table 2. Sample Selection Sensitivity Indicators for Face-on Disks

\begin{tabular}{ccccc}
\hline \hline $\begin{array}{c}\mu_{0}^{c} \\
\left(\mathrm{mag} \mathrm{arcsec}^{-2}\right)\end{array}$ & $\begin{array}{r}r_{s}(\min .) \\
(\operatorname{arcsec})\end{array}$ & $\Gamma(-17.5) / \Gamma_{\max }^{s}$ & $\begin{array}{c}h_{s}(-17.5) \\
(\mathrm{kpc})\end{array}$ & $\begin{array}{c}h_{s}\left(\Gamma_{\max }^{s}\right) \\
(\mathrm{kpc})\end{array}$ \\
\hline 25 & 90 & 0.33 & 6.1 & 18.9 \\
24 & 32 & 0.58 & 3.9 & 6.7 \\
23 & 20 & 0.58 & 2.4 & 4.2 \\
22 & 13 & 0.57 & 1.5 & 2.7 \\
21 & 8 & 0.60 & 1.0 & 1.6 \\
20 & 6 & 0.50 & 0.6 & 1.3 \\
19 & 5 & 0.37 & 0.4 & 1.1 \\
18 & 4.5 & 0.26 & 0.2 & 1.0 \\
\hline
\end{tabular}

